\title{
A secure road traffic congestion detection and notification concept based on V2I communications
}

\author{
Vinh-Thong Ta ${ }^{\mathrm{a}, *}$, Amit Dvir ${ }^{\mathrm{b}}$ \\ ${ }^{a}$ School of Physical Sciences and Computing, University of Central Lancashire, Preston, \\ PR1 2HE, UK \\ ${ }^{b}$ Ariel Cyber Innovation Center, Department of Computer Science, Ariel University, Ariel, \\ Israel
}

\begin{abstract}
Applying vehicular (V2X) communications in detecting traffic congestion is a promising approach, as smart and self-driving vehicles are equipped with sensors that can be used to detect an incident anywhere in real-time. Unfortunately, without appropriate security measures and careful design the communication can be vulnerable to malicious attacks, causing even more damage on the roads. Addressing these problems, we propose a high-level system architecture and a security protocol specifically designed for congestion detection based on vehicleto-infrastructure (V2I) type communication. The security properties of our proposed approach are formally verified using the ProVerif tool, and its efficiency compared to the traditional traffic light systems is demonstrated through simulations with the Veins framework. Results show that our system is secure against a large set of attacks, and can have lower total travelling time compared to three traditional traffic light approaches based on induction loop, lane area detector/camera (installed near the junctions), and static lights.
\end{abstract}

Keywords: Security, V2I communications, traffic congestion detection. 2010 MSC: 68M12

\section{Introduction}

Many studies on road traffic control problems address the efficiency aspect, for instance, proposing algorithms and methods for traffic lights to reduce congestion and $\mathrm{CO} 2$ emission (see, for example, the studies [1-12]). Besides the

5 theoretical research studies, adaptive traffic control systems are already widely deployed on the roads, using technologies of induction loop or cameras to mitigate congestion. For instance, SCATS [8] and SCOOT [9] measure traffic intensity by installing vehicles detector loops at the junctions, while InSync [10] relies on camcorders. Although adaptive traffic control systems mitigate traffic

${ }^{*}$ Corresponding author 
congestion, they are not capable of detecting any traffic congestion or incident taking place outside the range of the cameras or detector loops.

There are initiatives and studies, for example [13, 14], on using surveillance camera systems in smart cities to detect road congestion. Unfortunately, monitoring the entire roads, especially motorways, would require a huge number of 15 detector loops or cameras throughout the road, which can be expensive. Further, surveillance camera systems for this purpose can be less effective in bad weather with poor visibility. Nowadays, self-driving vehicles are equipped with LIDAR and multi camera systems (e.g. [15, 16]) that could be used to detect a road incident. However, V2X communication would be necessary to report this.

The security problems of traditional traffic light systems were examined in the past, for instance, in the studies [17-20, where the authors found that the communications in some early versions of traffic light systems happened without any encryption, as well as the default passwords remaining unchanged. More recently, researchers also found the possibility for generating fake traffic congestion in social navigation systems such as Waze [21.

Adapting vehicular (V2X) communications in traffic management is a promising approach to overcoming the limitations of traditional methods. However, this would increase the possibility for attacks as the attackers can intercept and modify the messages as well as compromise the vehicles and road-side units with so malware. Several projects investigated the security and privacy problems of vehicular (and intra-vehicular) communications such as SeVeCom 17 and EVITA ${ }^{2}$ The main goal of these projects is to secure the wireless vehicle-to-vehicle (V2V), vehicle-to-infrastructure (V2I) as well as the intra-vehicular communications, preventing attackers from causing accidents by eavesdropping and manipulat-

35 ing the communications or stealing the secret keys stored inside the on-board modules. The anonymity of vehicles on the road has been also investigated, and solutions proposed such as using pseudonym or group signature 22. These projects provided a generic concept of secure vehicular communications, which we adapted and modified for the road traffic control context and problems.

Addressing the above-mentioned problems, to fill the gap, we propose a secure road congestion detection and notification concept (namely, a system architecture and a secure communication protocol) based on the vehicle to infrastructure (V2I) type communication. The security of the proposed communication protocol against both the external and insider attackers is formally 45 proved with the ProVerif verification tool [23], and the efficiency of the proposed concept is demonstrated through simulations with the Veins framework [24. In our approach a vehicle would be able to identify the lane IDs based on the road information sent by the road side units.

We note that this paper is an improved version of our previous conference

\footnotetext{
${ }^{1}$ Secure vehicle communication project, 2006-2008, https://trimis.ec.europa.eu/project/securevehicle-communication

${ }^{2}$ E-safety vehicle intrusion protected applications project, 2008-2011, http://www.evitaproject.org/
} 
paper [25] with improved algorithms, security analysis and simulation. Section 2 highlights the related works. Section 3 discusses the adversary model, followed by our proposed smart traffic control concept based on V2I communications in Section 4. We provide a formal security analysis of our concept in Section 5 . and a simulation to demonstrate its efficiency against different traditional ap-

55 proaches in Section 6. Section 8 summarizes our observations and findings, and we conclude the paper in Section 9

\section{Related Works}

Zhou et. al. 2], proposed a decentralised traffic light control approach using wireless sensor network. Their system has a three-layer architecture that consists of a traffic flow policy model and a high-level coordination between the so-called intersection control agents. The authors in [3] proposed a real time vehicle detection and tracking system using surveillance videos. They proposed a video processing algorithm for vehicle detection and analysing traffic density on a particular lane. Unlike our V2I approach, video-based methods would require 65 a large amount of cameras to detect congestion on long roads/motorways.

Leontiadis et. al. [6] evaluated the effectiveness of a decentralized trafficbased navigation system in which instead of distributing traffic information centrally, vehicles report their current information such as location, speed, and travel time to their neighborhood. Their solution assumes vehicle-to-vehicle

7o $(\mathrm{V} 2 \mathrm{~V})$ communications and that each vehicle is able to act as a traffic sensor, to measure the surrounding traffic density (vehicle quantity), and then exchange the information in an ad-hoc manner. Their results show that a decentralized approach can greatly reduce congestion in a realistic scenario.

The above studies focused mainly on the effectiveness of traffic control sys-

75 tems, but not the security problems. From the security aspect, Jeske [17] presented a man-in-the-middle attack on GPS coordinate transmissions in order to trick navigation services into inferring non-existent congestion while in an article, Zetter [18, outlined how the wireless vehicle detection systems (an earlier version of Sensys Networks VDS240) deployed in 40 U.S. cities were vulnera-

so ble and could be exploited to generate false traffic congestion. The author, C. Cerrudo, found that those Sensys networks devices communicated with each other without any encryption or security mechanism, making it possible for an attacker to modify the messages to mislead the control system. Similarly, in his article, Grad [19] outlined that by adjusting the signal times at the key inter85 sections in Los Angeles, USA, insider attacks on command centers could also create massive delays.

A study from the University of Michigan in 2014 [20] points out that a large portion of traffic lights in the United States communicated with each other wireless over the $900 \mathrm{Mhz}$ and $5.8 \mathrm{Ghz}$ ISM band without any encryption. In

9o order to connect to the $5.8 \mathrm{Ghz}$ traffic signals, attackers only needed the SSID of the corresponding device. The second security hole the researchers found was that the passwords were left default, which could be found on the traffic light manufacturer's website. In addition to these, to gain access to the $900 \mathrm{Mhz}$ 
networks attackers also needed a 16-bit slave ID. The authors also found a vulnerability in the open debug port in the VxWorks OS which allowed the attacker to read-modify-write any memory register. In another attack (called remote keypad), the attacker could freeze the current intersection state, modify the signal timing, or change the state of any light. Fortunately, the hardware based malfunction management unit (MMU) would still be able to detect any 100 illegal states (conflicting green or yellow lights), and took over with the 4-way red flashing. In this case, a technician needed to manually reset the traffic signal to recover from the illegal state, the attacker could turn every intersection on the network into a 4-way stop, causing traffic congestion.

Laszka et al. 26] introduced a novel approach for evaluating vulnerabilities of transportation networks by identifying traffic signals that have the greatest impact on congestion. Therefore, those signals may be the targets of attacks. The authors showed that this is a NP-hard problem, so a polynomial-time heuristic algorithm for computing approximately optimal attacks was presented. Yin et al. 27 presented a threat of data spoofing over the U.S. Department of

${ }_{110}$ Transportation traffic control system. The data spoofing can be done from one single attack vehicle only, with the attack goal of creating traffic congestion. Some further vulnerabilities are discussed in [28].

Machine learning has been used recently to estimate or predict traffic congestion. In [29] the authors proposed an efficient traffic congestion estima-

115 tion method based on model-based estimation through virtual sensors using the Kalman filter. The procedure is based on the data collected from sensors such as induction loops and then applying the KNN classification to estimate congestion. The authors tested their method by deploying induction loops in the SR60 Highway of California to collect traffic data, and showed that their algorithm can estimate congestion with good accuracy. The authors in [30] discussed several methods between 2014-2019 on the application of machine learning to predict crowd flow (human mobility prediction), traffic flow and public transit flow (e.g. bike sharing and metro flows) on spatio-temporal datasets. They examined five groups of prediction approaches, including statistics-based, traditional machine learning-based and deep learning-based approaches.

Social networking concept has already been broadly applied to smart traffic control. Waz $\mathrm{H}^{3}$ is a community-based navigation application, where users can share traffic status on certain roads with each other, such as indicating traffic congestion, police presence, construction, etc. Researchers managed to exploit 130 a vulnerability in an earlier version of Waze [21, and misled the application causing fake traffic congestion. As a result, the application suggested an alternative longer route for the nearby drivers. Finally, camera-based products for smart city are also widely deployed and used in self-driving vehicles that could potentially be applied for traffic management (e.g. MobilEy $\rfloor^{4}$.

135 Unfortunately, only very few studies proposed specific protocols and concepts

\footnotetext{
${ }^{3}$ Waze, https://www.waze.com/en-GB/about

${ }^{4}$ MobilEye, https://www.mobileye.com
} 
for smart traffic control systems based on vehicular communications. The main difference between our work and the previous studies is that we propose an approach that combines those different areas, such as V2X communications, traffic management and security. The main focus of our work is the security design against different attacker models; however, we also show the effectiveness of our method compared to the traditional traffic management solutions based on induction loops, area lane detector or cameras.

\section{Our Adversary Model}

In our case, the main goal of the attackers is to mislead the traffic control system with incorrect or incomplete traffic data leading to inefficient traffic schedule. They can prevent normal light functionality, for instance, by setting all lights to red or green at the same time (e.g. [20]). Although recent traffic light systems are protected by a hardware-based malfunction management unit (MMU) that prevents inconsistent/unsafe light states avoiding accidents, this will not prevent the attackers from setting inefficient light programs (e.g. longer red time than required).

As for the attacker's ability, in our approach we consider the insider and external attackers, as well as a set of possible attack methods, as follows:

- Insider attackers are parts of the roads, such as compromised vehicles or road side units. They can be instructed to send incorrect, fake traffic messages to the other participants on the roads. The incorrect messages are encrypted or signed with the valid keys of the compromised nodes.

- External attackers are not parts of the road (i.e. they are neither compromised vehicles nor road side units), and hence, they do not possess any valid keys by default. In our case, the main goal of the external attackers is exploiting the design flaws in the communication and the security protocols. We assume that an external attacker is able to eavesdrop the entire wireless communication and modify, forge and create fake messages with the keys it possesses. Further, it can replay and relay any captured packets to any participant. This adversary model is strong as in vehicular ad-hoc networks attackers can normally only overhear certain parts of the roads, and hence their ability of intercepting and sending messages is more limited [31. Since we will prove that our proposed system is secure against the stronger attacker model, it is also secure against a weaker model.

Furthermore, we also consider the attacks based on their nature, as follows:

- Local attacks are attacks that require the attacker to have either physical or local access to the RSUs, controllers or vehicles. Local attack is a means to recruit compromised vehicles or RSUs.

- Remote attacks are attacks where the victims (e.g. vehicles, RSUs) are exploited and taken control over from anywhere. 
- Non-collusion attack: In this case, we are talking about a single attacker who does not cooperate with the other attackers to reach a certain goal.

- Collusion attack: In this case, the attackers cooperate with each other to reach the same goal. They can share resources and synchronize their activities. This includes the so-called Sybil attack [32] when a node uses several fake identities to mislead the traffic control systems.

The motivation of the attackers in our case includes causing false traffic congestion and unnecessary traffic diversion, like in [18]. Namely, the attackers' intent is to modify or fake the traffic data and then get it accepted by the controllers who are responsible for setting the traffic light programs. We consider the following cases where congestion can be caused if the attacker

1. achieves that the duration of the red light is prolonged on a busy road/lane segment, leading to a potential financial impact (e.g. 33);

2. achieves that certain roads (e.g. the road on which the attacker is travelling) have longer green light duration than normal, so that the attacker can benefit from this (e.g. [18]);

3 . achieves that the traffic flow on certain roads is diverted to other roads because of fake congestion reports (e.g. [21]).

\section{Proposed Secure Traffic Congestion Detection and Management Methodology}

We present our proposed concept for detecting and managing traffic congestion based on the so-called vehicular public key infrastructure (VPKI). As outlined in [31, asymmetric key cryptography seems to be the most suitable approach in securing vehicular communications as it has the potential to provide confidentiality, authenticity, privacy and accountability (e.g. for police investigation) at the same time. This concept has been applied in several previous studies and projects such as the SeVeCom project 22]. However, we modify it for the traffic congestion detection context that, to the best of our knowledge, has not been done before. Our system architecture, protocol and algorithms are different from the one in the project.

\subsection{System architecture}

The basic concept of our method is depicted in Fig. 1. In our approach, we define road segments as a part of the road from one junction to another junction with traffic lights, and denote them by $R_{i}, i \in\{1, \ldots, n\}$.

Each lane segment $L n_{i}$ is defined by a triple, $L n_{i}=\left(L i d_{i}^{j}, D_{i}^{j}, R S U_{i}^{n}\right)$, where $\operatorname{Lid}_{i}^{j}$ is the unique ID of a lane segment, $D_{i}^{j}$ is the direction of the lane that can be, for instance, north $(N)$, south $(S)$, east $(E)$, west $(W)$, or from the perspective of a vehicle it can be forward $(F W)$ and reverse $(R E V)$, as well as 215 a road side unit $(R S U)$ that is installed at the beginning of a lane segment. 

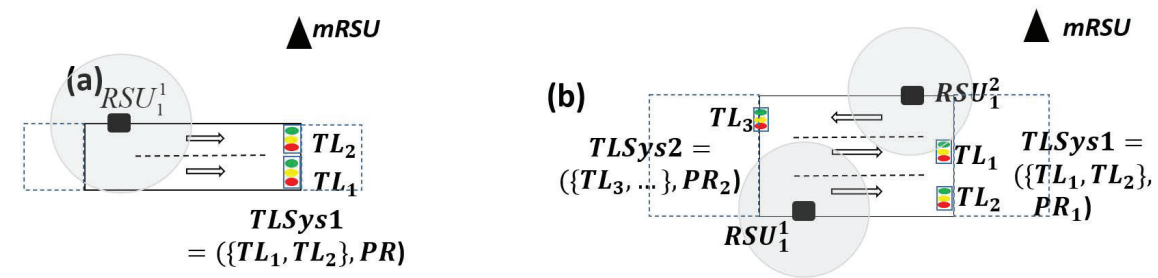

Figure 1: An architecture overview of the road and lane segments with the RSUs, mRSUs. Point (a) shows a road defined by $R_{1}=\left(\left\{\left(L i d_{1}^{j}, D_{1}^{j}, R S U_{1}^{1}\right), j=1,2\right\}, m R S U\right)$, and in (b), $R_{1}=\left(\left\{\left(L i d_{1}^{j}, D_{1}^{j}, R S U_{1}^{n}\right), j=1,2,3, n=1,2\right\}, m R S U\right)$.

Each road segment $R_{k}$ is defined by a set of lane segments, and a so-called main road-side unit (mRSU), namely, $R_{k}=\left(\operatorname{Rid}_{k},\left\{L n_{j}\right\}\right.$, mRSU). One mRSU is installed in a region of several road segments and junction(s).

At a junction, we define the so-called traffic light system, denoted by TLSys, which includes a set of traffic lights $\left(\left\{\mathrm{TL}_{1}, \ldots, \mathrm{TL}_{M}\right\}\right)$ for some natural $M$, and the program $(P R)$ that controls those lights. Formally, a region (denoted by $\left.R e g_{n}\right)$ can be defined as $\operatorname{Reg}_{n}=\left(\left\{R_{k}\right\},\left\{T L S y s_{j}\right\}, m R S U\right)$, where $\left\{R_{k}\right\}$ and $\left\{T L S y s_{j}\right\}$ are a set of road segments and a set of traffic light systems, respectively, in this region, while mRSU is the main RSU installed in this region and is responsible for changing the traffic light programs in $\left\{T L S y s_{j}\right\}$.

Fig. 1/ (a) highlights a simple road segment containing two lane segments, and a traffic light system, TLSys1, with two lights $T L_{1}, T L_{2}$, and a default light program, $P R$. A RSU $\left(R S U_{1}^{1}\right)$ is installed at the start of the road segment that covers both lanes. Finally, mRSU is the main road side unit assigned to this

230 region. Fig. 1 (b) depicts a road segment and three lane segments with two RSUs. The same approach is applied for the bigger road segments.

The RSUs should be installed close to the traffic lights/junctions, at the start of the lane segments in order to inform the incoming vehicles about the lane segments they are about to enter. We assume that each RSU has a short-range radio antenna that enables transmission of data within the radius equal to the width of some lanes. With the short-range antennas we would like to minimise the number of messages that a vehicle has to deal with.

\subsubsection{Architectural requirements}

Our approach includes a few architectural requirements regarding the vehicles, the RSUs and mRSUs, such as:

- For vehicles: Each vehicle has a built-in satellite navigation system (SatNav/GNSS), e.g. GPS, Galileo, GLONASS, or BeiDou.

Vehicles are equipped with a V2X capable internal module (IM) that stores the addresses of the mRSUs in a record (denoted by MRSUrecord). The format of MRSUrecord is defined in Table 1. 
Table 1: The record MRSUrecord for some $N \geq 1$

$M R S U r e c o r d=\left\{1 . m R S U_{1}: R e_{1}, 2 . m R S U_{2}: R e_{2}, \ldots, N . m R S U_{N}: R e_{N}\right\}$

Each row of MRSUrecord contains the ID of a mRSU and the region $\left(R e_{j}\right)$ in which this mRSU is installed, which is specified by SatNav coordinates.

To reduce the size of MRSUrecord, only the information about the mRSUs inside a country/state is stored, where a vehicle was registered. This can be updated with new mRSUs when a vehicle arrived in a new country/state. Alternatively, this record can be built into the SatNav system used inside the vehicles.

In addition, an IM is responsible for handling non-sensitive cryptographic operations with the public keys (e.g., signature verification or asymmetric encryption). An IM is connected to a sensor (or to the odo/mileo-meter) and records the distance that a vehicle traveled within a road segment.

A vehicle is equipped with a good quality camera (or a set of cameras), with which it can take pictures about the road segment it arrived at, and the IM module is capable of extracting and differentiating the lanes from the pictures taken, as well as identifying the lane in which the vehicle is currently traveling (e.g. see 34]).

In addition to the IM module, each vehicle is equipped with a hardware security module (HSM) or a trusted platform module (TPM) that is responsible for performing security critical operations such as digital signature generation as well as the storage of the private keys. We will discuss further the choice between TPM and HSM in Section 8

- For RSUs: Each RSU is equipped with a trusted platform module (TPM) for digital signature generation and storing the signature keys. In addition, they are installed with an internal module that is responsible for putting together and broadcasting messages for the vehicles.

- For mRSUs: Each mRSU is equipped with an internal module (IM) that can receive and verify the traffic status messages from the vehicles. An IM stores a record, Roadrecord, about the road segments in the region where the mRSU is installed, and the lane information in each road segment, such as lane IDs, number of lanes, lane and pavement width.

In order to broadcast road information for the vehicles to help them with identifying the lane they are traveling on, at the installation time each RSU stores a record called RoadInfo about the road segment where it has been installed. Specifically, the format of RoadInfo is defined in Table 2. 
Table 2: The format of RoadInfo for some NumLanes $\geq 1$

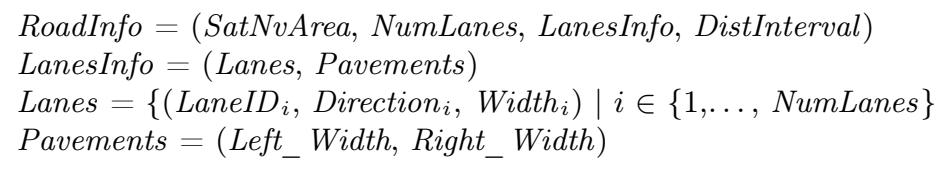

RoadInfo contains the SatNav area of the road segment (SatNvArea), the number of lanes in the road (NumLanes), a set of lanes information (LanesInfo), and a distance interval DistInterval. LanesInfo includes information about the lanes and pavements in the road segment. Namely, Lanes contains the lane ID, the lane direction, and the width of the lanes. From the perspective of

285 the arriving vehicles, $i=1$ is the leftmost lane while $i=$ NumLanes is the rightmost, or vice versa. Pavements contains the width of the left and right pavements (zero in case of no pavement). Finally, DistInterval is the interval defined by two distances from the start of the road segment, during which vehicles are required to notify if they have not got stuck until that point. This interval depends on the length of the road segment. The road information can be edited at the installation time of the RSU, as normally, information about the road does not change often. In case of any change due to construction, the corresponding RoadInfo is updated accordingly.

\subsection{Cryptographic Keys}

As mentioned, we assume a public key infrastructure for vehicular systems, where communications between the vehicles and RSUs, mRSUs are digitally signed, and the certificate authority/authorities (CA) is responsible for issuing and revoking certificates for the public keys. Following the concept of SeVeCom 22, 31, 35, the vehicles have a pair of long-term public and private keys. These

300 are generated by and stored inside the HSM or TMP in the vehicle at the time of registration or manufacture. This way nobody can read or obtain the private key. The long-term public key part is certified by the CA (there can be several CAs for different regions and countries). Each vehicle is also given a unique long-term identity and the public key of the CA at the registration time.

To prevent the vehicles from being tracked in the long term, short-term key pairs are used in the communications instead of the long-term key pairs. This kind of solution is referred to as "pseudonymous authentication" [22, 35. The $\mathrm{CA}$ is given the authority to link long-term identities with several corresponding short-term credentials to provide accountability. The short-term public keys do not reveal the vehicles long-term identity, and each vehicle will switch to another (not previously used) short-term key pair at a junction. Junctions prevent the observer/attacker from tracking a certain vehicle due to the crowd, and to the fact that messages signed under different short-term private keys cannot be 
Table 3: The variables and their values/calculations in the algorithms

\begin{tabular}{|c|c|}
\hline Variable & The calculation/value of the variable \\
\hline 1. RSUBroadcast $=$ & (RSUid, MRSUid, RoadInfo, curr time) \\
\hline 2. signed_RSUBroadcast $=$ & Sign(RSUBroadcast, $\left.S K_{R S U}\right)$ \\
\hline 3. curr $\operatorname{loc}=$ & (currLaneID, dist, loc) \\
\hline 4. notstuck_msg $=$ & $\begin{array}{l}\text { ("Not Stuck", lanes_info, curr time, } \\
\text { curr_loc, curr_state, mRSUid) }\end{array}$ \\
\hline 5. signed_notstuck_msg $=$ & $\operatorname{Sign}\left(\right.$ notstuck $\left.\operatorname{msg}, S K_{V E}^{S}\right)$ \\
\hline 6. lanes info $=$ & signed_RSUBroadcast $[$ RoadInfo $][$ LanesInfo $]$ \\
\hline 7. taken picset $=$ & enhancePics(takePics () , lanes info) \\
\hline 8. currLaneID $=$ & idenCurrLaneID(taken_picset, lanes_info) \\
\hline 9. dist $=$ & updateDistance() \\
\hline 10. state $=$ & updateState(loc) \\
\hline 11. loc_of stuck $=$ & (currLaneID, dist, loc) \\
\hline 12. stuck_msg $=$ & $\begin{array}{l}\text { ("Got Stuck", lanes_info, loc of stuck, } \\
\text { curr time, state, Vtype, mR } \overline{\text { SUid) }}\end{array}$ \\
\hline 13. signed_stuck_msg $=$ & $\operatorname{Sign}\left(\right.$ stuck $\left.\operatorname{msg}, S K_{V E}^{S}\right)$ \\
\hline 14. goagain $\mathrm{msg}=$ & ("Go Again", loc_of stuck, curr time) \\
\hline 15. signed_goagain_msg $=$ & Sign(goagain_msg, $S K_{V E}^{S}$ ) \\
\hline 16. newSKKE $=$ & $\mathbf{S K}()$ \\
\hline 17. newP $\frac{V E}{S E}=$ & $\mathbf{P K}\left(\right.$ newS $\left.K_{V E}^{S}\right)$ \\
\hline 18. $\operatorname{loc}=$ & updateSatNavLoc () \\
\hline 19. curr time $=$ & updateTime() \\
\hline 20. cert_request_msg $=$ & $\left(I D_{V E}, C A i d\right.$, newPK$K_{V E}^{S}$, loc, curr time $)$ \\
\hline 21. signed_request $=$ & Sign(cert_request_msg, $S K_{V E}^{L}$ ) \\
\hline 22. encrypted_signed_request $=$ & AEnc(signed_request, $P K_{C A}^{E n c}$ ) \\
\hline 23. mRSUid $=$ & updateMRSÜ(loc, MRSUrecord) \\
\hline 24. no br_alarm $=$ & ("No Br", loc, curr time, mRSUid) \\
\hline 25. signed_no_br $=$ & Sign(no_br_alarm, $S K_{V E}^{S}$ ) \\
\hline 26. cert_new_key $=$ & $\left(\right.$ CAid, ID $\overline{D V E}_{V E}$, newCert ${ }_{V E}^{S}$, curr_time $)$ \\
\hline 27. signed cert $=$ & Sign(cert_new key, $\left.\left.S K_{C A}^{L}\right)\right)$ \\
\hline 28. encrypted_signed_cert $=$ & AEnc(signed_cert, $P K_{V E}^{E n c}$ ) \\
\hline 29. notstuck time $=$ & signed_notstuck_msg[curr_time] \\
\hline 30. notstuck_curr_loc $=$ & signed_notstuck_msg[curr_loc] \\
\hline 31. notstuck loc $=$ & curr loc [loc] \\
\hline 32. notstuck_state $=$ & signed_notstuck_msg[curr_state] \\
\hline 33. $\mathrm{XPK}_{V E}^{\mathrm{S}}=$ & $\operatorname{Cert}\left(P K_{V E}^{S}\right)\left[P \bar{K}_{V E}^{S}\right]$ \\
\hline 34. got $\operatorname{roadID}=$ & getRoadID(loc, Roadrecord) \\
\hline 35. got_lanes_info $=$ & getLanes(got_roadID) \\
\hline 36. stuck_time $=$ & signed_stuck_msg[curr_time] \\
\hline 37. stuck curr loc $=$ & signed_stuck_msg[curr_loc] \\
\hline 38. stuck_loc $=$ & curr_loc[loc] \\
\hline 39. stuck_state $=$ & signed_stuck_msg[curr_state] \\
\hline 40. goagain_loc $=$ & signed_goagain_msg[loc_of_stuck $]$ \\
\hline 41. SetTLSys $=$ & $\left\{\operatorname{TLSys}_{1}, \ldots, \overline{\operatorname{TLSys}}_{N}\right\}$ \\
\hline 42. SetCV $=$ & $\left\{\mathrm{CV}\left(L_{1}\right), \ldots, \mathrm{CV}\left(L_{M}\right)\right\}$ \\
\hline
\end{tabular}

linked. New short-term key pairs are generated by the HSM/TPM inside the 315 vehicles. The private keys are kept inside the HSM/TPM or stored encrypted inside the vehicle, while the public key parts are certified by the CA. 
Table 4: Notations Used in The Algorithms (Constant values/records Part 1)

\begin{tabular}{cc}
\hline \hline Notations & Explanation \\
\hline RSUid/MRSUid & Long-term ID of an RSU/main RSU \\
\hline CA/CAid & The certificate authority/its long-term ID \\
\hline Vtype & A vehicle type (e.g., "emergency", "bus") \\
\hline RoadInfo & (SatNavLoc, NumLanes, LaneInfo, DistInterval) \\
\hline LaneInfo & (Lanes, Pavements) \\
\hline SatNvLoc & The SatNav area/coord. of a road segment \\
\hline NumLanes & A set of properties about the \\
\hline Lanes & lanes in a road segment \\
\hline Pavements & Contains the width of the pavements \\
& in a road segment \\
\hline DistInterval & An interval of values of distance from the road start \\
\hline Roadrecord & A record of all the road segments in a region \\
\hline Initime & An initialisation time value \\
\hline Resendlimit & A limit value for resending a message \\
\hline Current_time & The current time \\
\hline Current_loc & The current SatNav location of a vehicle \\
\hline$\triangle T_{R S U}$ & A time period after which a RSU broadcasts \\
\hline$\triangle T_{\text {stuck }}^{\text {thres }}$ & A threshold after which a "Got Stuck" alarm is sent \\
\hline$\triangle T_{\text {goagain }}^{\text {hres }}$ & A threshold after which a "Go Again" msg is sent \\
\hline$\triangle T_{\text {resend }}$ & A Threshold after which a msg is re-sent \\
\hline$\triangle T_{\text {fresh }}$ & A time threshold to check message freshness \\
\hline$\triangle T_{\text {notstuck }}$ & A threshold after which a "Not Stuck" msg is sent \\
\hline$\triangle T_{\text {signal }}^{\text {apdate }}$ & A threshold until there is no satellite signal \\
\hline$\triangle T_{\text {light }}^{\text {update }}$ & A threshold after which the congestion states are checked \\
\hline
\end{tabular}

\subsection{The Secure Traffic Congestion Detection Procedure}

As discussed in the previous section, the RSUs are installed at the start of the lane segments in order to broadcast the record RoadInfo to the approaching vehicles so that they know which lane of a road segment they arrived at. The SatNav/GNSS system built in a vehicle is used to identify roads, junctions and a larger region with several road segments, but cannot be used to identify a single lane accurately [36.

For better readability, we keep the description of the algorithms as simple 325 and short as possible, and refer the reader to Table 3 for the values or calculation of the variables used in the algorithms. In the algorithms, we use generic, implementation specific parameters and variables rather than proposing specific values. We examined some specific values in simulation (Section 6).

Algorithm 1 defines the operation of a RSU. After each $\triangle T_{R S U}$ time period, a RSU broadcasts a digitally signed message, signed RSUBroadcast, along with the certificate of the public key of $\operatorname{RSU}\left(\operatorname{Cert}\left(P \bar{K}_{R S U}^{\bar{L}}\right)\right)$. As shown in row 1 of Table 3, signed_RSUBroadcast contains the ID of the RSU (RSUid), the ID of the main RSU for that region (mRSUid), the record RoadInfo and the timestamp (curr_time) for message freshness.

Algorithm 2, $\overline{\mathrm{VE}}$, outlines the behaviour of a vehicle. When VE receives a broadcast message from a RSU (line 3), it carries out several verification steps 
Table 5: Notations Used in The Algorithms (Constant values/record Part 2)

\begin{tabular}{|c|c|}
\hline Notations & Explanation \\
\hline $\mathrm{VE} / I D_{V E}$ & A vehicle VE/its long-term ID \\
\hline$P K^{L} / P K^{S}$ & Long/short-term public (signature) key \\
\hline$S K^{L} / S K^{S}$ & Long/short-term private (signature) key \\
\hline$P K^{E n c} / S K^{E n c}$ & Long-term encryption public/private key \\
\hline $\operatorname{Cert}(P K)$ & The digital certificate of the public key $P K$ \\
\hline CurrLaneID & The current lane ID of a vehicle \\
\hline MRSUrecord & The record of the mRSUs and their regions \\
\hline Msg[e1] & The element e1 in the message Msg \\
\hline$V_{\text {ano }}^{\text {thres }}$ & $\begin{array}{l}\text { A threshold on the amount of the "not stuck" } \\
\text { vehicles, above which "anomaly" is reported. }\end{array}$ \\
\hline$\overline{D_{\text {ano }}}$ & $\begin{array}{l}\text { Anomaly check will be done within } D_{a n o} \\
\text { distance before and after the location of the stuck }\end{array}$ \\
\hline $\bar{T}$ ano & $\begin{array}{l}\text { Anomaly check will be done within } T_{\text {ano }} \\
\text { time before and after the time of the stuck. }\end{array}$ \\
\hline$T L$ & An ID of a traffic light \\
\hline TLSys & $\begin{array}{l}\text { An ID of the traffic light system at a junction } \\
\quad\left(\text { TLSys }=\left\{\mathrm{TL}_{1}, \ldots, \mathrm{TL}_{n}\right\} \text { for some } n\right)\end{array}$ \\
\hline SetTLSys & $\begin{array}{c}\text { A set of traffic light systems } \\
\left(\text { SetSTLSys }^{\prime}=\left\{\operatorname{TLSys}_{1}, \ldots, \text { TLSys }_{n}\right\} \text { for some } n\right)\end{array}$ \\
\hline $\mathrm{PR}_{\text {Default }}$ & The default program of a traffic light system. \\
\hline $\mathrm{PR}_{i}$ & A program of a traffic light system for some $i$. \\
\hline$\triangle T_{T L}$ & A threshold after which a $\mathrm{PR}_{i}$ is updated \\
\hline SetPR & $\begin{array}{l}\text { The set of all pre-defined programs of all } \\
\text { the traffic light systems in a region. }\end{array}$ \\
\hline $\mathrm{ST}_{j}$ & $\begin{array}{l}\text { A state of a traffic light system for some } j \text {. } \\
\text { e.g. for TLSys }=\left\{\mathrm{TL}_{1}, \mathrm{TL}_{2}, \mathrm{TL}_{3}, \mathrm{TL}_{4}\right\} \text { the } \\
\text { state Green-Red-Green-Red refers to the color } \\
\text { of the lights } \mathrm{TL}_{1}, \ldots, \mathrm{TL}_{4} \text {, respectively. }\end{array}$ \\
\hline M & $\begin{array}{c}\text { A pre-defined value to specify that the green light } \\
\text { time in one light state is } M \text { times longer than } \\
\text { in another light state. }\end{array}$ \\
\hline SetST & $\begin{array}{l}\text { The set of all pre-defined states of all } \\
\text { the traffic light systems in a region. }\end{array}$ \\
\hline
\end{tabular}

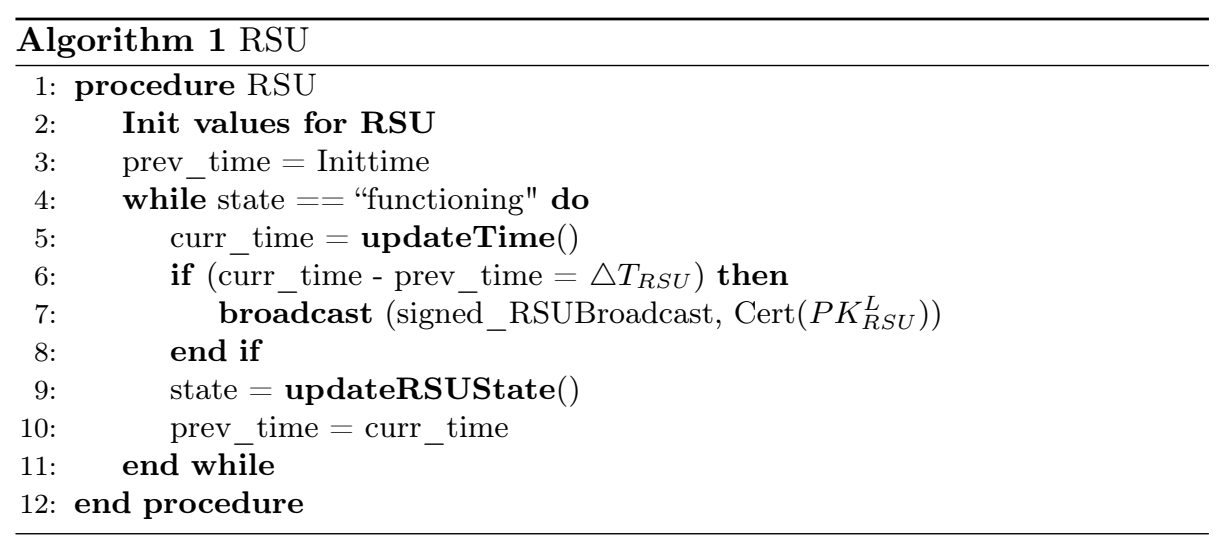


Table 6: Notations Used in The Algorithms (Variables)

\begin{tabular}{cc}
\hline \hline Notations & Explanation \\
\hline dist & $\begin{array}{c}\text { The distance a vehicle travels } \\
\text { since entering a new road segment }\end{array}$ \\
\hline speed & Captures the current speed of VE (see getSpeed()) \\
\hline lanes_info & Captures the received lane properties \\
\hline taken_picset & Captures a set of pictures taken by a vehicle \\
\hline enh_picset & Captures a set of enhanced pictures of a vehicle \\
\hline pic_set & Captures a set of pictures from a RSU \\
\hline currLaneID & Captures a current lane ID of a vehicle \\
\hline roadID & Captures an ID of a road segment \\
\hline got_roadID & An ID of a road segment fetched by a mRSU \\
from its road record
\end{tabular}

(line 4). First, it checks the validity of the public key of the RSU in the attached certificate $\left(\operatorname{Cert}\left(P K_{R S U}^{L}\right)\right)$, and then, the validity of the signature. Afterwards, VE checks if the time stamp in the message is fresh (i.e., the difference between the current time and the received timestamp is within a threshold $\triangle T_{\text {fresh }}$ ). In case of success, and the satellite signal is unavailable or weak (line 5), VE continues its operation as defined in procedure VEONROAD.

Otherwise, if the satellite signal is good in the area (line 7, Alg. 2), then VE checks if the received MRSUid (signed_RSUBroadcast/MRSUid]) is the same as the mRSU ID the vehicle stores in its MRSUrecord (line 8) ${ }^{5}$ In line 9, VE compares its current SatNav location (loc, row 18 of Table 3) with the SatNav area in the received broadcast, signed_RSUBroadcast[RoadInfo][SatNvArea]. In case they are consistent (VE is inside the correct road segment), VE continues its operation as defined in procedure VEONROAD (line 10, Alg. 2p.

In procedure VEONROAD, first, VE resets the distance value dist and sets its state to "onroad" (lines 18-20). Then, until the vehicle VE leaves the road segment (i.e. state $==$ "leave"), in case its speed is less than a given threshold, $S$, for a certain period of time, $\triangle T_{\text {stuck }}^{\text {thres }}$, it sends a "Got Stuck" message to the

\footnotetext{
${ }^{5}$ Although a vehicle can fetch the mRSU ID from its MRSUrecord, it requires the availability of a satellite signal. To prepare for the case when there is no/weak signal in an area, the mRSU ID is included in a RSU broadcast. Besides, with this redundancy, a vehicle can also detect compromised RSUs that intentionally send wrong/fake mRSU IDs.
} 
Table 7: Notations Used Inside The Algorithms (Functions/Activities)

\begin{tabular}{|c|c|}
\hline Notations & Explanation \\
\hline Sign(Msg, SK) & The signature of Msg with the key $S K$ \\
\hline AEnc(Msg, PK) & The encryption of Msg with the key $P K$ \\
\hline broadcast Msg & Broadcasts a message Msg \\
\hline $\mathbf{S K}()$ & Returns a new secret key, newSK \\
\hline PK(newSK) & Returns a new public key for newSK \\
\hline receive Msg & Receive a message Msg \\
\hline delete Msg & Delete a message Msg \\
\hline verif(Msg) & $\begin{array}{l}\text { The verification of the message Msg } \\
\text { returns true if successful }\end{array}$ \\
\hline updateTime () & Returns the current time \\
\hline updateSatNavLoc () & Returns the current satnav location \\
\hline updateState(loc) & Returns the current state of a vehicle \\
\hline getSpeed () & Returns the current speed of a vehicle \\
\hline $\begin{array}{l}\text { numNotStuckVeh } \\
\left(\mathrm{R}, \mathrm{L}, \mathrm{T}, \mathrm{D}, \mathrm{T}^{\prime}\right)\end{array}$ & $\begin{array}{l}\text { Returns the number of NOT stuck vehicles } \\
\text { within a distance } \mathrm{d} \text { from the location } \mathrm{L} \text {, } \\
\text { and within the time } \mathrm{T} \text { ' from the time } \mathrm{T} \text {, } \\
\text { based on the set of records } \mathrm{R} \text {. }\end{array}$ \\
\hline $\begin{array}{c}\text { enhancePics } \\
\text { (pic_set, lanes_info) }\end{array}$ & $\begin{array}{l}\text { Returns a set of enhanced pictures of the } \\
\text { input set of pictures based on a } \\
\text { set of lanes properties }\end{array}$ \\
\hline $\begin{array}{l}\text { drawLanes } \\
\text { (pic set, lanes info) }\end{array}$ & $\begin{array}{l}\text { Draw virtual lanes lines in the pictures } \\
\text { in pic set based on a set lanes info }\end{array}$ \\
\hline $\begin{array}{l}\text { idenCurrLaneID } \\
\text { (taken_picset, lanes_info) }\end{array}$ & $\begin{array}{l}\text { Returns the current lane ID based on } \\
\text { a set of taken pictures and lane info }\end{array}$ \\
\hline takePics () & Returns a set of taken pictures \\
\hline getRoadID(loc, Roadrecord) & $\begin{array}{c}\text { Returns the ID of a road segment based } \\
\text { on the SatNav location, and } \\
\text { a record of road segments }\end{array}$ \\
\hline getLanes(roadID) & Returns the lanes of a road segment \\
\hline $\mathbf{C V}(\mathrm{L})$ & $\begin{array}{l}\text { Returns the congestion value on the } \\
\text { lane L (e.g., number of stuck cars) }\end{array}$ \\
\hline \begin{tabular}{l|l} 
Func1 & Func2
\end{tabular} & Func1 and Func2 are running in parallel \\
\hline setLightProg(TLSys, PR) & $\begin{array}{c}\text { Set the program of the traffic light } \\
\text { system TLSys to PR }\end{array}$ \\
\hline CurrProg(TLSys) & $\begin{array}{l}\text { Returns the current program (PR) of } \\
\text { the traffic light system TLSys }\end{array}$ \\
\hline
\end{tabular}

mRSU installed in this region (lines 22-24). When VE manages to go again (i.e. 355 speed $>S$ ) for at least a pre-defined time period, $\triangle T_{\text {goagain }}^{\text {thres }}$, a signed "Go Again" message is sent to the same mRSU that was previously sent the corresponding "Got Stuck" message (lines 25-26). The signed "Go Again" message will also be sent (even if $\triangle T_{\text {goagain }}^{\text {thres }}$ has not elapsed) when VE leaves the road segment where it got stuck (lines 32-33).

If a vehicle $\mathrm{VE}$ does not get stuck from the point it received a RSU broadcast when entering a road segment up to a random distance in DistInterval, it sends the so-called "Not Stuck" message once to the corresponding mRSU (lines 2729). This is to make the mRSU capable of detecting anomalies in case the compromised vehicles intentionally send fake "Got Stuck" messages.

When VE leaves a road segment and reaches a junction, it starts updating 


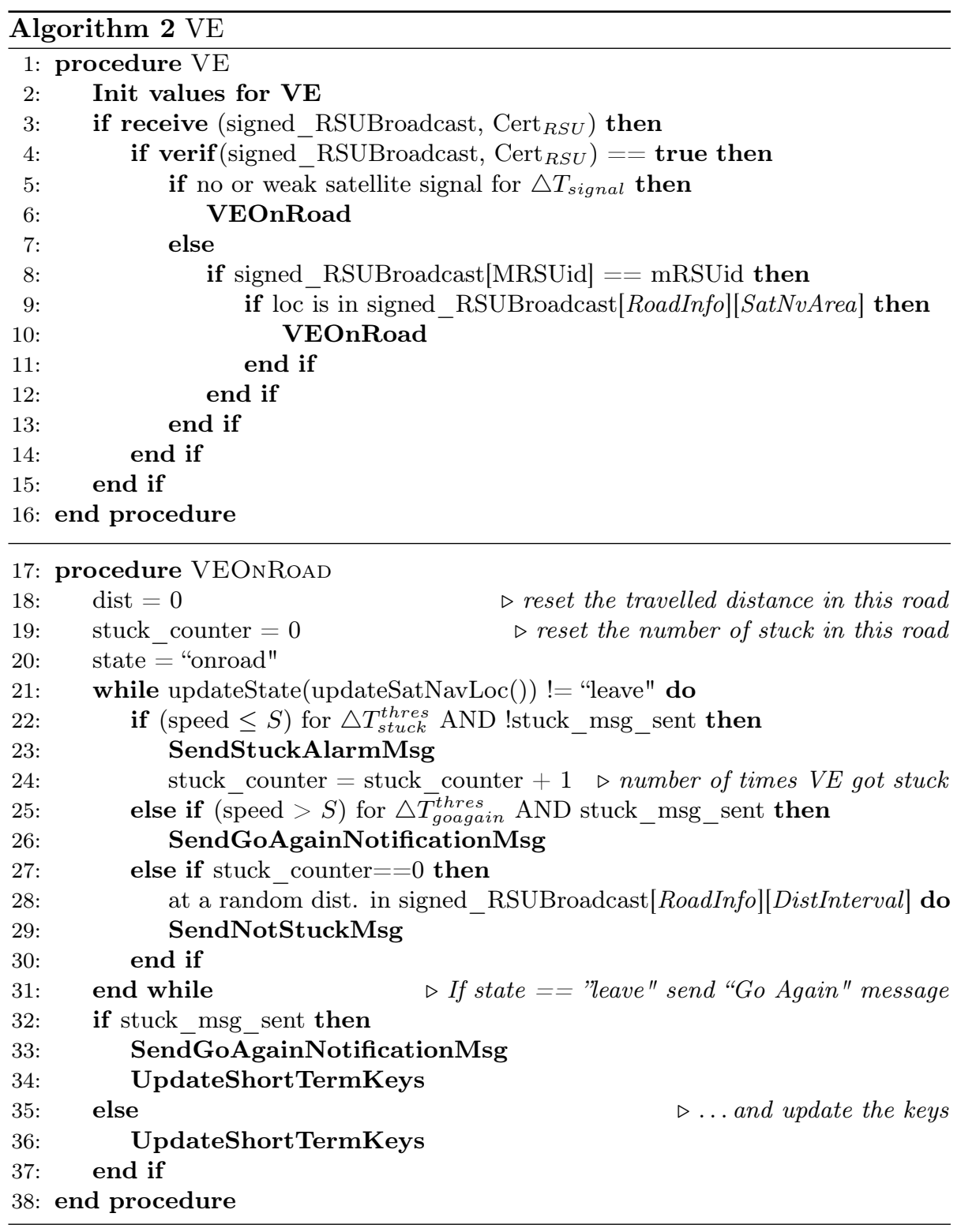




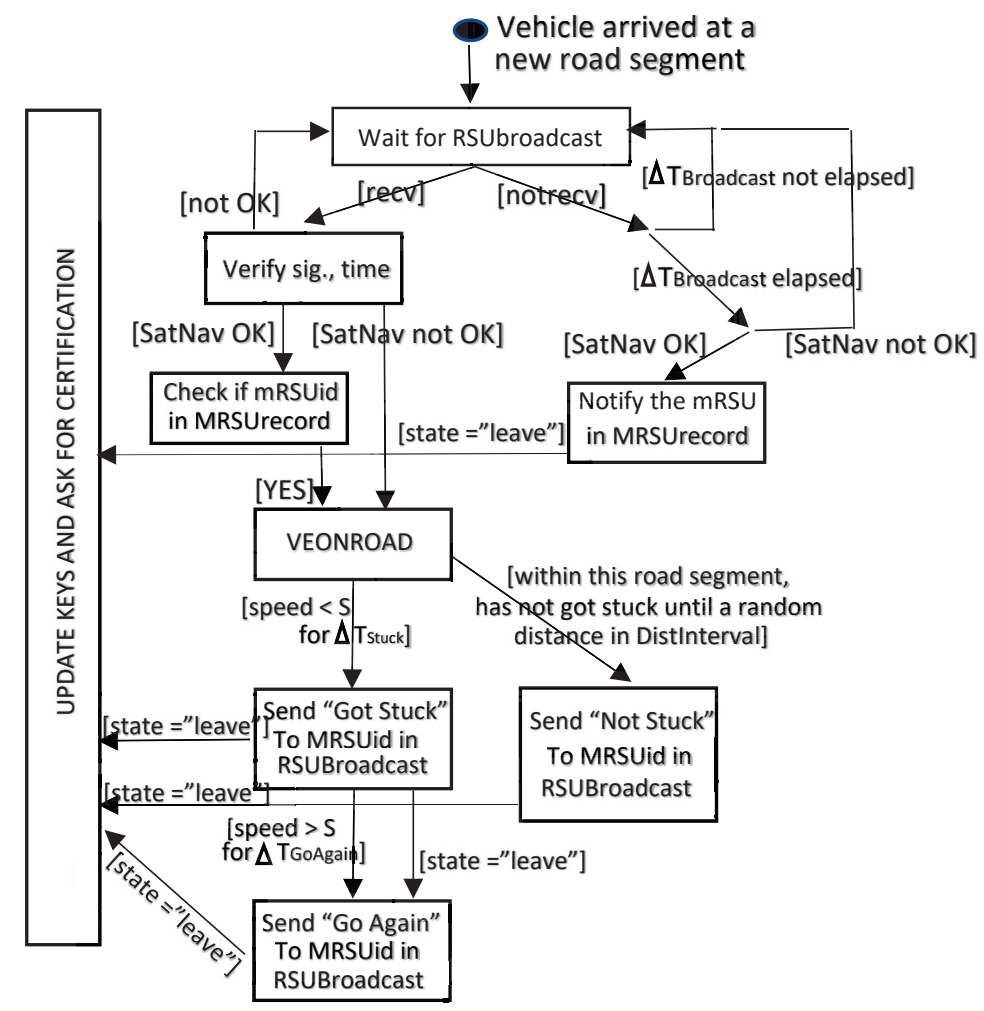

Figure 2: The operation of a vehicle (VE) when arrived at a new road segment.

the new short-term signature key pairs (lines 34 and 36, procedure VEOnroad). The rationale behind this is to achieve a higher degree of anonymity for $\mathrm{VE}$, as this area is where we expect a large number of vehicles change their short-term keys at the same time, making it more difficult to (re)-identify a vehicle after 370 that ${ }^{6}$ Although this concept would allow vehicles to be traceable within a single road segment, the long-term tracking is more difficult [37.

Algorithm 3 defines how VE sends a message. In line 2, the "Got Stuck" message, is signed using the actual short-term private key of VE (signed_stuck_msg), and as shown in rows 12-13 of Table 3 , contains

1. the "Got Stuck" tag;

2. the information about the lanes, lanes_info, which VE received from the RSU (see row 6 of Table 3);

3. the location where VE got stuck (loc_got_stuck, row 11 of Table 3), con-

${ }^{6}$ This solution is less effective in case there are only few vehicles on the roads. (Asymmetric) Encryption could be used to achieve confidentiality all time, but it would increase the computation and message overhead. 


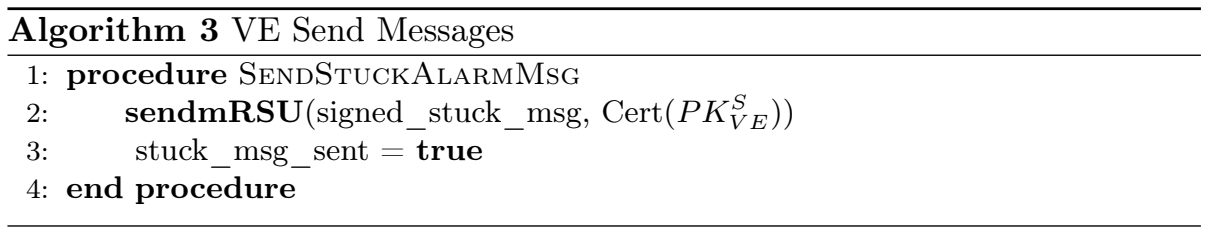

5: procedure SendGoAgainNotificationMsg

6: $\quad$ sendmRSU(signed_goagain_msg, $\left.\operatorname{Cert}\left(P K_{V E}^{S}\right)\right)$

7: $\quad$ stuck_msg_sent $=$ false

8: end procedure

9: procedure SENDNotStuckMsG

10: $\quad$ sendmRSU(signed_notstuck_msg, $\left.\operatorname{Cert}\left(P K_{V E}^{S}\right)\right)$

11: end procedure

380 at a junction. In line 3, a new short-term secret key is generated based on the function SK, then the corresponding public key is derived from the new private key using the function PK (line 4). In line 5, an encrypted and signed request, encrypted_signed_request, along with the certificate of the long-term public (signature) key of VE is sent to the CA. As shown in row 20 of Table 3 . the message cert_request_msg contains:

1. the long-term ID of VE and the ID of the CA,

2. the newly generated short-term public key $\left(\right.$ new $\left.P K_{V E}^{S}\right)$, for which VE requests the certificate, and

3. the current SatNav location of VE (loc), and the current time (curr_time).

The new short-term private key $\left(n e w S K_{V E}^{S}\right)$ is kept in secret inside the HSM/TPM module of VE. As row 21 of Table 3 shows, this message is signed 


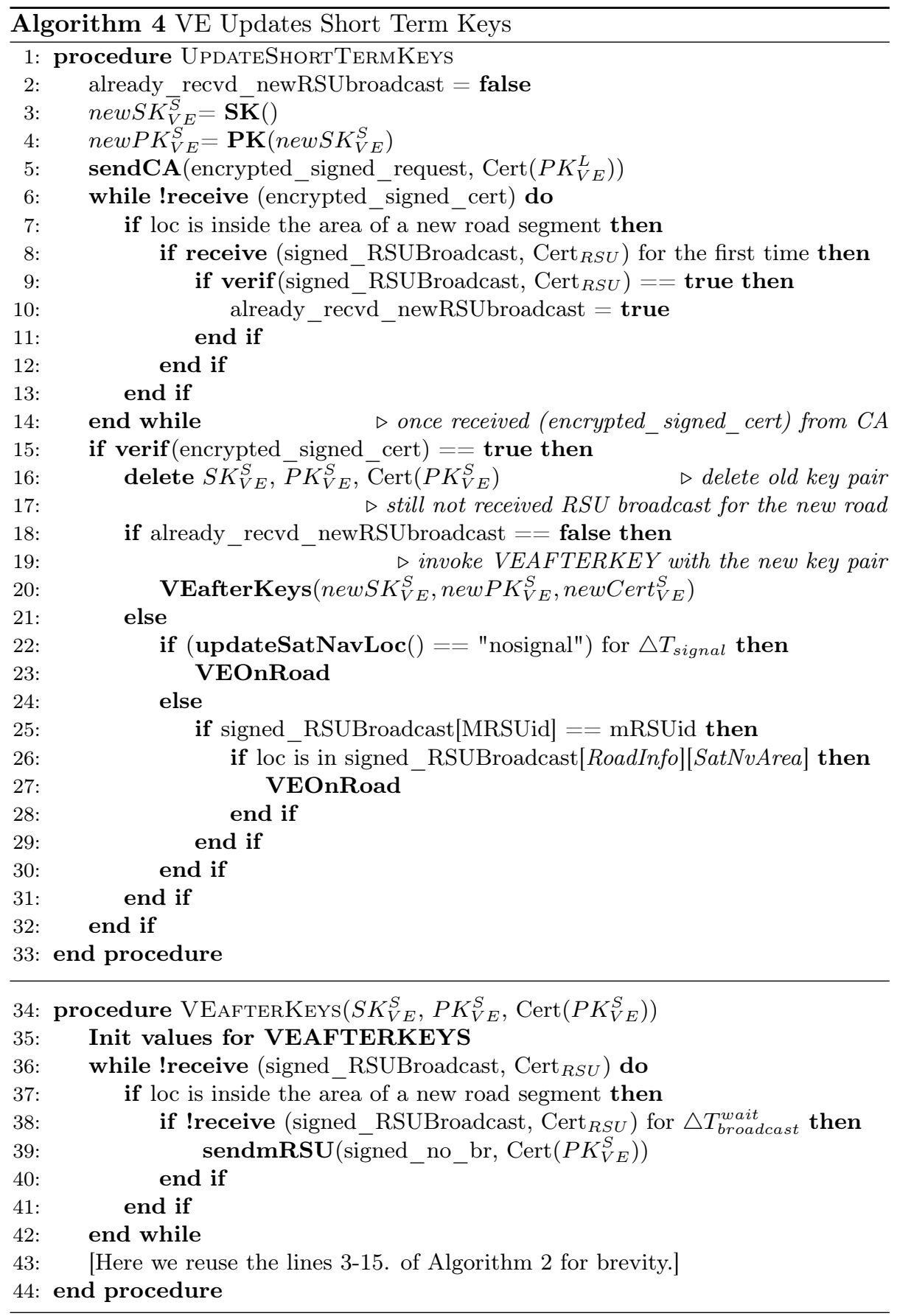


using the long-term signature private key of VE $\left(S K_{V E}^{L}\right)$, which is then encrypted using the (long-term) encryption public key of the CA $\left(P K_{C A}^{E n c}\right)$ in row 22 . The encryption is necessary to keep the long-term ID $\left(I D_{V E}\right)$ confidential to avoid the vehicle being tracked for a long time. For security purpose, different algorithms are used for signature and encryption.

Lines 6-14 of Algorithm 4 capture the case when VE, while waiting for the certificate of its new public key, enters a new road segment. If $\mathrm{VE}$ receives a

415 RSU broadcast in this road segment (line 8), after a successful verification (line 9 ), it sets the flag "already received a new broadcast", and saves this broadcast (line 10). Later, when VE receives the key certification from the CA, after a successful verification steps (line 15), VE deletes the old short-term keys to free space and enhance its anonymity (line 16). In case VE has not received 420 any RSU broadcast for the new road segment yet (line 18), it continues its operation with the new short-term keys and certificate as defined in lines 3444. Otherwise, depending on the availability of the satellite signal in that area, it verifies the received mRSU ID against its MRSUrecord (lines 25-26), and continues as procedure VEONROAD (line 23 and 27).

Procedure VEafterKeys defines an operation of VE after the key certification from the CA. If VE has not received any RSU broadcast from the new road for a $\triangle T_{\text {broadcast }}^{\text {wait }}$ time period, then $\mathrm{VE}$ sends to the corresponding mRSU a socalled "no broadcast" message, signed_no_br, indicating a potential problem with the RSU. Upon receiving this, the $\overline{m R S U}$ notifies the authority so the 430 broken/compromised RSU can be fixed. The message signed no br, as shown in rows 24-25 of Table 3, contains the tag "No Br", the current SatNav location and time, and the mRSUid fetched from the MRSUrecord (row 23 of Table 3).

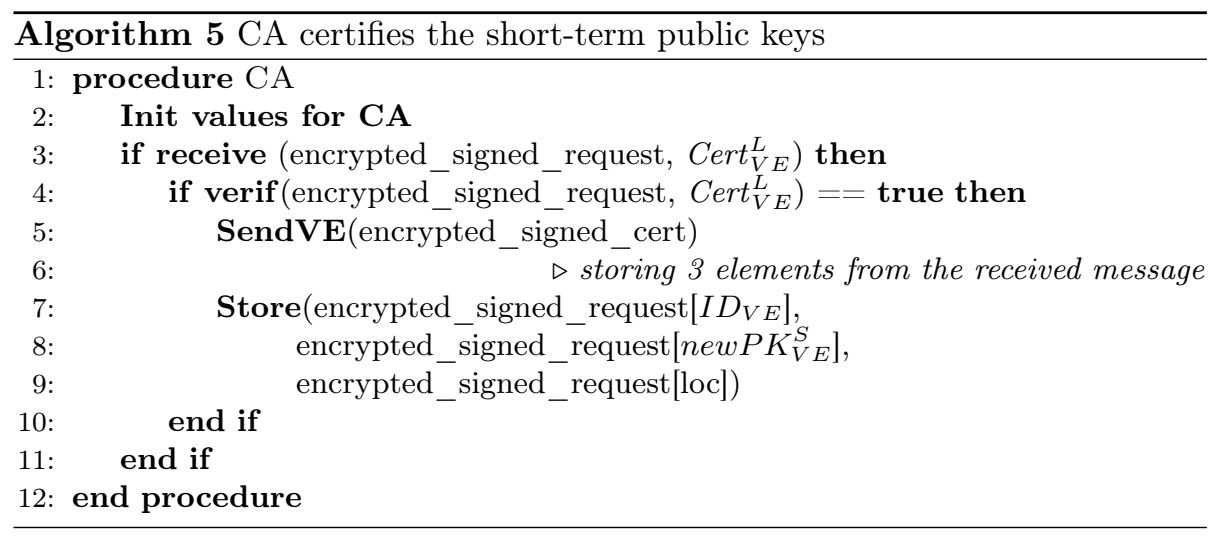

In Algorithm 5 , once the $\mathrm{CA}$ receives a certificate request message (line 3), in line 4 it decrypts the message with its own decryption private key, then verifies 435 the signature and checks whether the time stamp is fresh. Once successful, the CA checks if CAid is its own ID, then, in line 5 it sends back to VE a message that contains the certification for the new short term public key. Again, this message is signed with the private key of the CA, and then encrypted using 
the encryption public key of VE. The CA stores the long-term ID of VE, the new short-term public key and the SatNav location of VE for accountability purposes, for example, during an investigation of a criminal incident (lines 7-9).

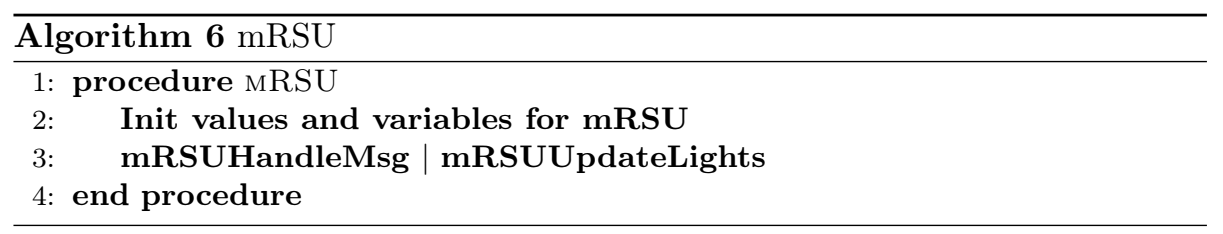

A mRSU, in Algorithm 6, performs more computation tasks than a RSU, including signature verification, as well as modifying the phases or states of the traffic lights under its control (region). The operation of a mRSU consists of two functions running in parallel, mRSUHandleMsg specifies how a mRSU handles a received message, while mRSUUpdateLights specifies how a mRSU regularly checks and updates the traffic light programs based on the congestion values.

In Algorithm 7 ( $m R S U H a n d l e M s g$ ), we distinguish among the cases when a mRSU receives a "Not Stuck" message from a vehicle (line 3), a "Got Stuck" so message (line 5) or a "Go Again" message (line 7).

In the first case (lines 11-17), mRSU performs a verification including the validity of the signature and certificate, and the freshness of the timestamp (line 12). Next, the mRSU checks if the lanes information it stores about the road (got_lanes_info) is consistent with the received lanes information (line 455 13). In order to do this, as shown in rows 34-35 of Table 3 the mRSU extracts the road ID from its stored Roadrecord based on the received SatNav location (row 34), then reads the lanes information it stores about this road (row 35) to compare with the received one. In case of consistency, a so called "Not Stuck" entry with the timestamp, the location, the state and the public key inside the ${ }_{460}$ received message is (temporarily) cached/stored in the storage of the mRSU called recordset (line 14, Alg. 7). This verification is for security purposes, to detect if a compromised RSU sent incorrect lane information to the vehicle.

Once a signed "Got Stuck" message (lines 18-27, Alg. 7) has been received from VE, and in case this message has not been stored before (line 19), the 465 verification of the message will take place. In line $20, \mathrm{mRSU}$ checks whether it is the addressee (i.e., mRSUid is correct), and the attached public key certificate and the signature on the message are valid, as well as if the timestamp is fresh. Finally, in line 21, the mRSU double checks if the lane information the vehicle sent is correct. In case the verification is successful, in line 22 , an entry with the

470 flag "stuck" is added into recordset. Finally, in line 23 the congestion values of the lane segments are updated with the flag "stuck" indicating that the congestion value is updated after receiving a "Got Stuck" message (see Algorithm 8).

When a mRSU receives a "Go Again" message (lines 28-35, Alg. 7), this message should be related to a previous "Got Stuck" message from the same 475 vehicle, indicating that a vehicle manages to go again after it got stuck. This relation is verified in line 29 , checking if there is already a corresponding "stuck" entry in the record record $\left(\mathrm{XPK}_{V E}^{S}\right)$. Thereafter, further verification will be done 


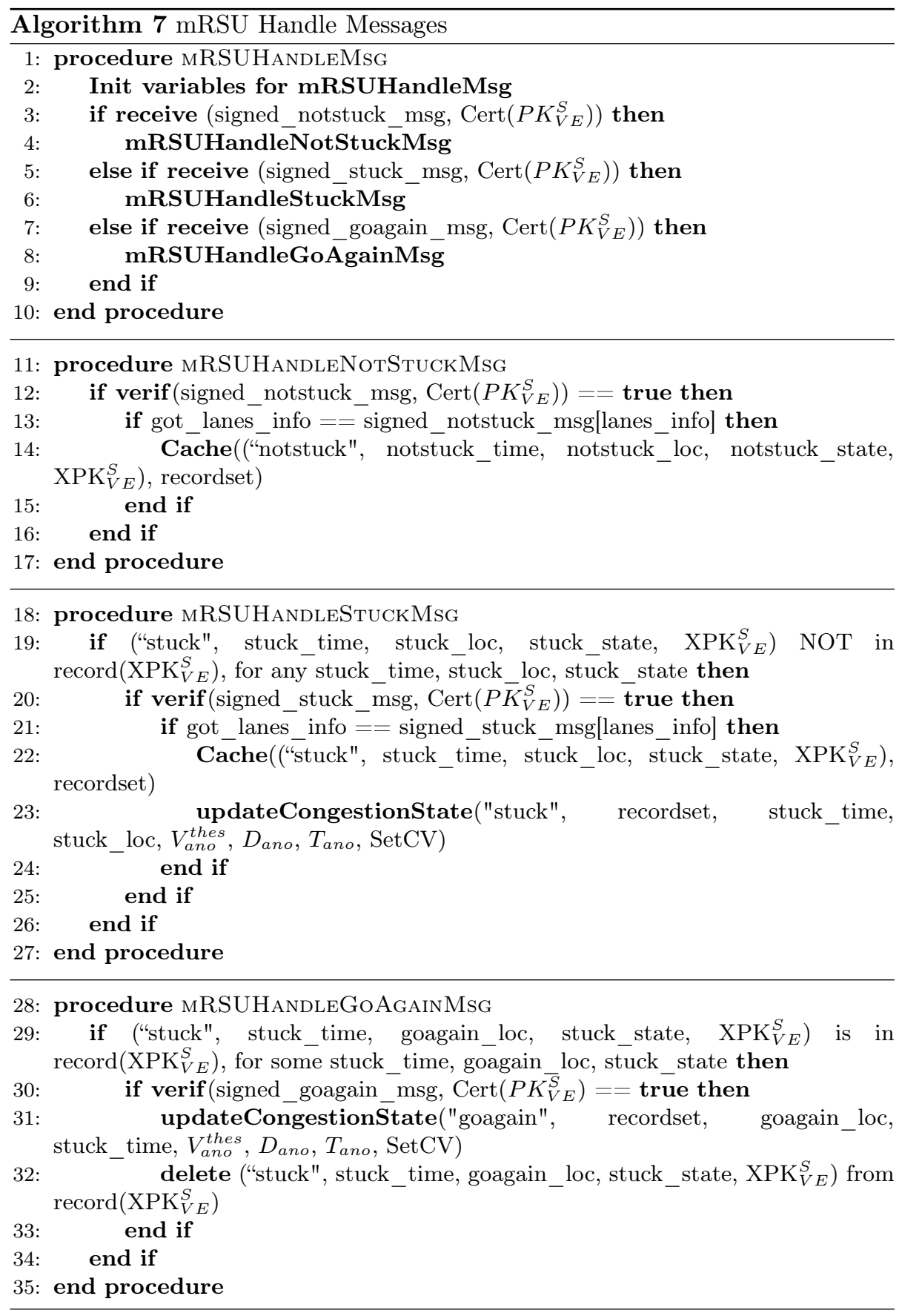


in line 30 and the congestion state will be updated, in line 31. Eventually, the "stuck" entry of $\mathrm{XPK}_{V E}^{S}$ will be deleted as the vehicle managed to go again.

The procedure updateCongestionState (Algorithm 8) expects as input the parameter flag, the set recordset, the location and the time of the stuck (loc and time) included in the received message, the threshold $\mathrm{V}_{\text {ano }}^{\text {thres }}$, the pre-defined values $D_{a n o}$ and $T_{a n o}$, and the set SetCV (see Tables 4.5 for the meaning of the notations). If a "Got Stuck" message is received (line 2 ) and no anomaly is

485 detected (line 4), then the congestion value (CV) of the lane where the vehicle got stuck (lane currLaneID) is increased. Otherwise, if a "Go Again" message is received, then the congestion value of the lane is decreased. Lines 3 and 11 double check whether the lane segment with the ID loc[currLaneID] (i.e., currLaneID inside loc) is in the area under the control of the mRSU.

Lines 15-18 (Algorithm 8) define how a mRSU updates the programs of the traffic light systems under its control area. After every pre-defined delay $\triangle T_{\text {lights }}^{\text {update }}$, a mRSU checks the congestion values of the lane segments in its area and updates the programs of the light systems accordingly (in lines 19-39, procedure UPDATELIGHTS).

495 Our main goal is to decrease the total travelling time of all the vehicles, hence, we compare the total congestion values of the crossing roads at the junctions. In line 20, for each traffic light system TLSys under control of the mRSU, given two sets of crossing lane segments $\left(\mathrm{L}_{1}, \ldots, \mathrm{L}_{n}\right.$ and $\left.\mathrm{L}_{n+1}, \ldots, \mathrm{L}_{k}\right)$ that follow the opposite light colours, the total congestion value of the two sets of lane segments 500 will be compared with each other (lines 24 and 30), and an appropriate program will be set, which favours one or another sets of lane segments (lines 26 and 32). PR1 is the program that gives green for the lane segments $\mathrm{L}_{1}, \ldots, \mathrm{L}_{n}$, while PR2 for $\mathrm{L}_{n+1}, \ldots, \mathrm{L}_{k}$. In lines 27 and 33 , after prolonging the green time for $\left(\mathrm{L}_{n+1}, \ldots, \mathrm{L}_{k}\right)$ and $\left(\mathrm{L}_{1}, \ldots, \mathrm{L}_{n}\right)$, respectively, the mRSU checks if a diversion ${ }_{505}$ of the traffic is needed (CheckIfDiversionNeeded). In case there is a set of lanes, denoted by LaneSameDir, with the same directions (LaneSameDir $\subseteq\left\{\mathrm{L}_{n+1}\right.$, $\left.\ldots, \mathrm{L}_{k}\right\}$ or $\left\{\mathrm{L}_{1}, \ldots, \mathrm{L}_{n}\right\}$ ) in which all lanes are blocked (full road blockage), or the total congestion values of those lanes do not decrease for $\triangle T_{D i v}$ time period then the traffic arriving in these lanes will be diverted (if possible), and the 510 authority will be automatically notified to resolve this. Otherwise, the default program $P R_{\text {Default }}$ will be set at the junction (line 36 ).

Note that the specific approaches used in the procedures increaseValue, decreaseValue, and the specific values in Tables 4 H5 are not defined in this paper. Instead we leave them in a generic form and carry out the simulation (in 515 Seciton 6) on some example specific values and approaches for the functions.

Algorithm 9 deals with the anomaly detection done by a mRSU. Basically, in line 2, the amount of vehicles sent "Not Stuck" (NS) within a pre-defined distance $D_{\text {ano }}$ before and after the location of the stuck (loc) and a pre-defined time period $T_{\text {ano }}$ before and after the time of the "got stuck" message is derived

${ }_{520}$ from recordset. If NS is larger than a pre-defined threshold value $V_{\text {ano }}^{\text {thres }}$ (line 3 ), then an anomaly is detected (i.e., true is returned). Intuitively, this means that the more vehicles notify the mRSU that they do not get stuck the more likely that there is an attack carried out by compromised vehicles. 


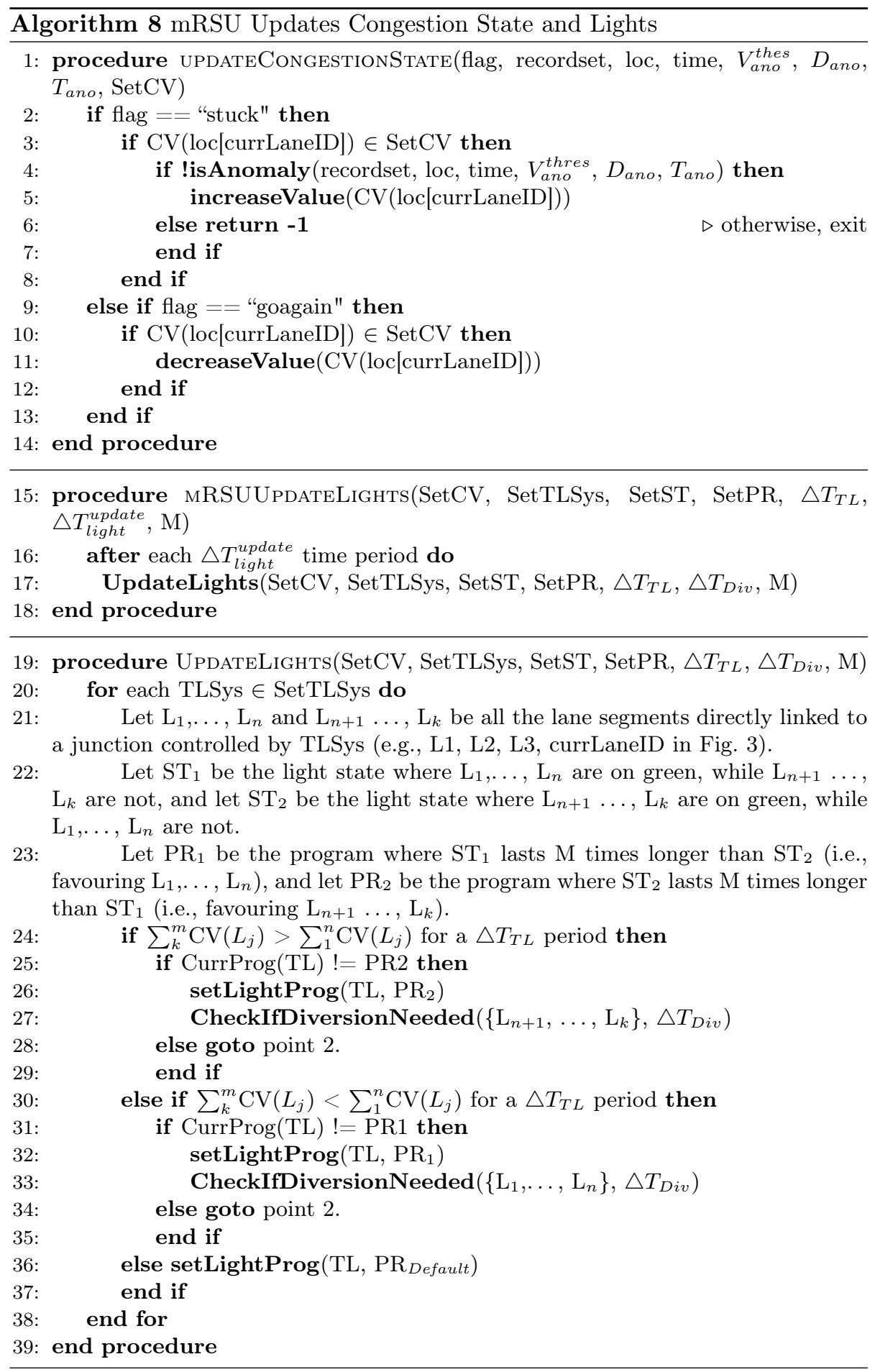



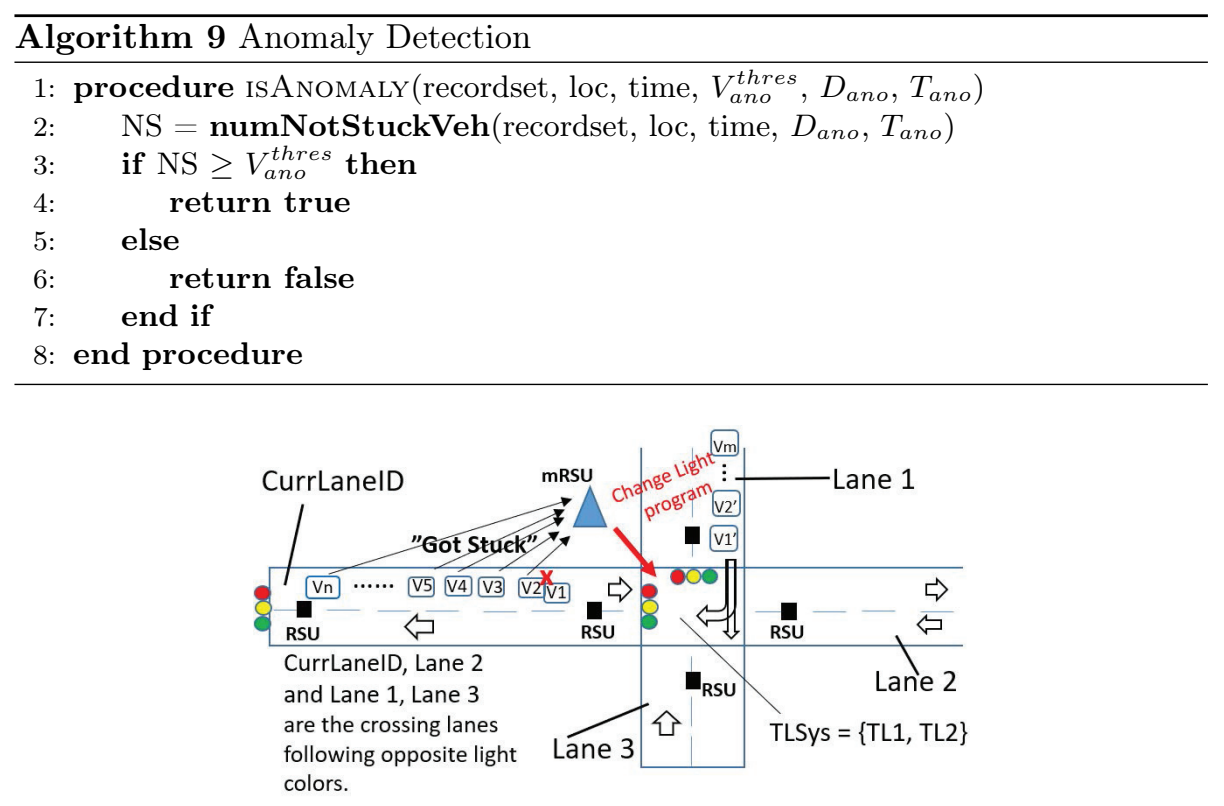

Figure 3: There is an accident caused by V1 and V2 in the lane NewLaneID, and all the vehicles behind them got stuck. \{CurrLaneID, Lane 2\} and \{Lane 1, Lane 3\} are the two sets of crossing lanes following the opposite light colors. The vehicles from Lane 1 can turn to right preventing $V 3, \ldots, V n$ in overtaking V1/V2 in the opposite lane.

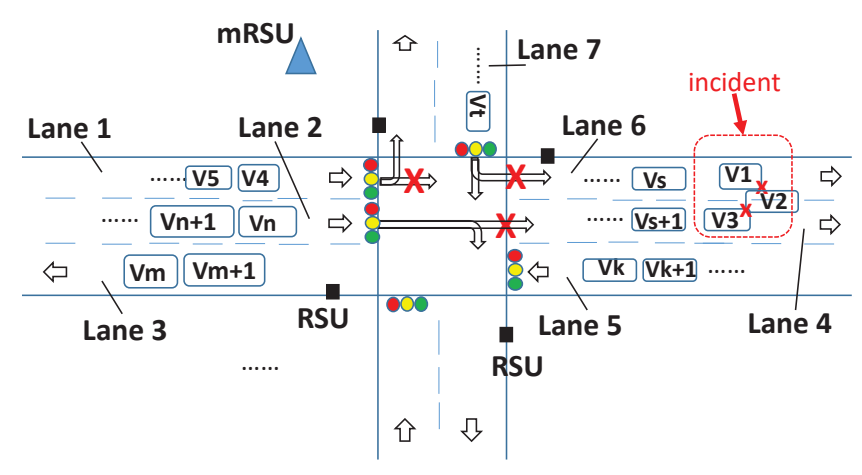

Figure 4: An accident scenario involving V1, V2, V3 where both the two lanes, Lane 4, Lane 6 of the same direction are blocked for more than $\triangle T_{D i v}$ time. The mRSU diverts the traffic from Lane 1, Lane 2, and Lane 7.

\section{Security Analysis}

We discuss a security analysis of our proposed protocol against the attacker models and attack methods defined in Section 3. Our analysis is based on the ProVerif tool [23], which has been extensively applied in verifying the security 
of different protocols (e.g. [38 40]). It assumes the attacker model in which the attacker can intercept, replay and relay as well as creating fake messages using the data it has and take part in different protocol sessions running in parallel.

\subsection{The security against external attackers}

For the external attackers who are not part of the system, we aim to analyze the key secrecy, authenticity and integrity properties of the protocol as three of the most relevant security properties, namely:

- Key secrecy: Here we formally prove the secrecy of the long-term and short-term private keys of the participants and long-term ID of the vehicles. Specifically,

1. the attacker cannot obtain the long-term private key $\left(\mathrm{SK}_{C A}^{e n c}, \mathrm{SK}_{V E}^{e n c}\right)$ for the asymmetric encryption used in the communication between a vehicle and the CA for certifying the new short term keys;

2. the attacker cannot obtain the short-term private key $\mathrm{SK}_{V E}^{S}$ used for signing the traffic messages ("Not Stuck", "Got Stuck" and "Go Again");

3. the attacker cannot obtain the long-term private keys $\mathrm{SK}_{V E}^{L}, \mathrm{SK}_{R S U}^{L}$, $\mathrm{SK}_{m R S U}^{L}$ and $\mathrm{SK}_{C A}^{L}$;

4. the attacker cannot obtain the long-term ID $\left(\operatorname{ID}_{V E}\right)$ of the vehicles.

- Authenticity: We prove three different properties for authenticity.

1. Property Auth1: In the communication between a vehicle VE and the CA to certify the new short-term keys (lines 34, 36 in Alg. 2 and Alg. 5), whenever VE finished the session and believes that it ran the session with the $\mathrm{CA}$, then this is really the case, and vice versa. Hence, an external attacker cannot impersonate either VE or the CA, and cannot generate valid signatures in the name of $\mathrm{VE}$ or $\mathrm{CA}$.

2. Property Auth2: Whenever a vehicle receives and accepts a broadcast message about the road segment (lines 3-4 of Alg. 2), the message was really generated freshly by a RSU.

3. Property Auth3: Whenever a mRSU accepts a ("Not Stuck", "Got Stuck" and "Go Again") message of a vehicle VE and accepts this as valid (i.e., the verification was successful), this message was really generated by VE, who sent it freshly.

- Integrity: We prove three different properties for integrity, as follows. Any modification of the messages broadcast by a RSU can be detected (Property Int1), any modification of the messages sent by a vehicle to a mRSU can be detected (Int2), and any modification of the messages exchanged between a vehicle and the CA can be detected (Int3).

To analyse the security properties of our system, we define a ProVerif process for each participant, namely, procRSU, procMRSU, procVE, and procCA. The protocol definition in ProVerif is as follows: 


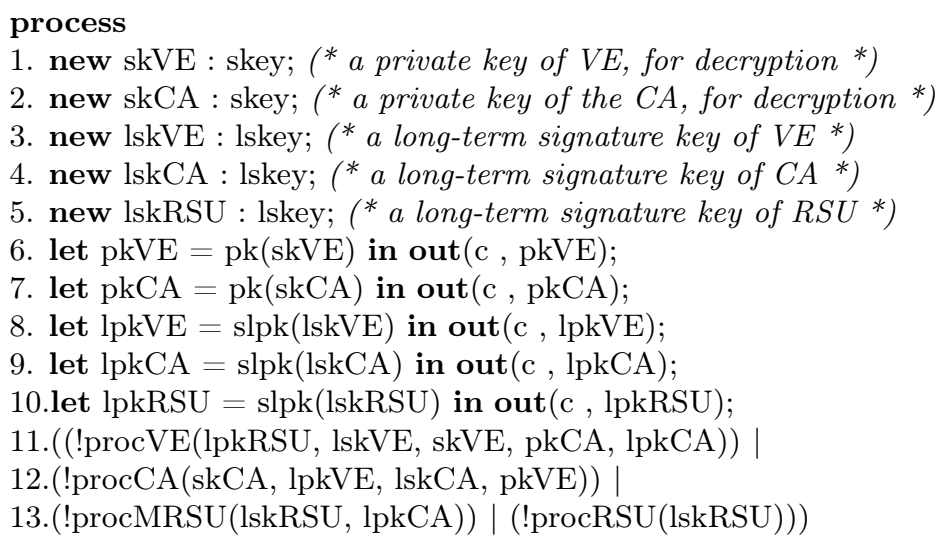

The keyword process denotes the main process (i.e., the protocol itself) and

570 ization of the long-term keys at the beginning, which will be used throughout the protocol. Specifically, line 1 (similarly, lines 2-5) defines the process that creates the data with the name $s k V E$ and of the type decryption private key (skey). Line 6 (similarly, 7-10) defines the process that computes the public key $p k V E$ from the secret key skVE using the function pk(skey), and sends it out on the channel $c$. Finally, lines 11-13 define the four processes proc VE, proc $C A$, procMRSU, procRSU running in parallel (denoted by "|"). The syntax element "!" means that each process is allowed to replicate its operation infinitely in order to capture parallel protocol sessions. We define the long-term and the

s8o short-term signing and encryption keys, and each process has parameters of the keys that it uses during the operation. The short-term signature keys of the vehicles ( $\operatorname{roc} V E$ ) are generated during the protocol run (not at the initialization) inside the process proc $V E$.

In ProVerif, the participants communicate via channels. We define a channel c, namely, "free c : channel.", allowing the attacker to overhear and intercept the communication. We define message types, such as the type for data (bitstring), type for long-term signature private and public keys (lskey, lpkey, respectively), and short-term signature private and public keys (sskey, spkey, respectively), the time (time) and road information (RoadInfo).

590 Then, we define cryptographic functions and primitives, such as the function for generating a public key from the corresponding private key, asymmetric key encryption (fun aenc(bitstring, pkey) : bitstring.) and decryption, digital signature generation with a short-term and long-term keys (fun ssign(bitstring, sskey) : bitstring., and fun sign(bitstring, lskey) : bitstring.) and verification.

The security properties are defined as follows in the ProVerif syntax. For the key secrecy properties:

1. To verify that the attacker cannot obtain the long-term private keys $\mathrm{SK}_{C A}^{e n c}$ and $\mathrm{SK}_{V E}^{e n c}$ for decryption, we specify two queries, namely "query secret skCA." and "query secret skVE.". 
2. For the short-term signature private key $S_{V E}^{S}$ of a vehicle: "query secret sskVE.".

3. For the long-term signature private keys $\mathrm{SK}_{V E}^{L}, \mathrm{SK}_{R S U}^{L}, \mathrm{SK}_{m R S U}^{L}, \mathrm{SK}_{C A}^{L}$ of VE, RSU, mRSU, CA, respectively: "query secret lskVE.", "query secret lskRSU.", "query secret lskMRSU.", and "query secret lskCA.".

4. For the long-term ID of VE ( $\left.\operatorname{ID}_{V E}\right)$ : "query attacker(IDve) ' 7

With these queries we can check if the attacker can obtain the keys and the long-term ID of VE. After running the verification, as result we got "RESULT not attacker(IDve/]) is true" for IDve, and "RESULT secret $x$ is true" for all the six private keys, where $x \in\{s k C A[/$, skVE[], sskVE[], lskVE[/, lskRSU/], $\left.{ }_{610} l s k C A / /\right\}$. This formally proves that the attacker cannot obtain any of these keys and the long-term ID of VE during the protocol runs.

For the authenticity properties, in ProVerif events and the so-called correspondence assertions are used (see sections 3.2.2-3.2.3 of the manual [41]).

For property Auth1, we define the following events:

1. event acceptsVECA(spkey,id).

3. event acceptsCA(spkey,id)
2. event termVECA(spkey,id).

4. event termCA(spkey,id).

The first event captures that a vehicle VE accepted running a protocol session with the CA and with the short-term public key to be certified (of type spkey) and with the long-term ID of VE (of type id). The second captures that VE believes that it terminated the protocol with the CA with the key of type spkey and the long-term ID of type id. The third and fourth events specify that the CA accepted, finished the protocol with the key of type spkey and with the long-term ID of type id, respectively. The correspondence assertions are:

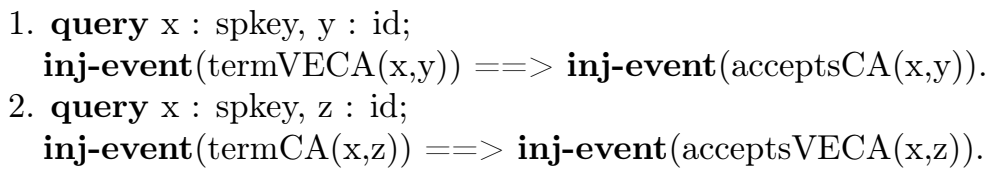

The first query instructs ProVerif to check if whenever a vehicle VE believes that it terminated a protocol session with the CA with a short-term signature public key $(x)$ and the long-term ID of VE $(y)$, then previously at some point, the CA accepted running this protocol session with a vehicle using the same short-term public key $(x)$ and the same long-term ID $(y)$. Here, we rely on the so-called injective correspondence (inj-event, see section 3.2 .3 of the manual [41]), in order to check the possibility of someone replaying the messages of the CA to VE. Similarly, the second query verifies that whenever the CA finished its session and sent back the certified short-term public key $x$ to a vehicle of

\footnotetext{
${ }^{7}$ Note that this property at the same time also proves the secrecy of keys $\mathrm{SK}_{C A}^{e n c}$ and $\mathrm{SK}_{V E}^{e n c}$ as they are used for decrypting the message containing IDve (lines 6-9 of Alg. 4 and lines 7-10 of Alg. 5p.
} 
long-term ID $y$, there was a vehicle with $y$ that accepted running this session with the $\mathrm{CA}$, and requested the certification for the key $x$.

For property Auth2, we define the following events:

1. event receivedVERSU(id, time, roadinfo). 2. sentRSU(id, time, roadinfo).

The first event captures that the vehicle VE received a broadcast containing an ID (of type id) of the RSU who sent it, a timestamp (of type time) and the record RoadInfo (of type roadinfo). The second event captures when a RSU sent the broadcast message that contains its ID (of type id), the current time (of type time) and a RoadInfo record. The correspondence assertions are:

$$
\begin{aligned}
& \text { 1. query } x: \text { id, } y: \text { time, } z: \text { roadinfo; } \\
& \text { event }(\operatorname{acceptedVERSU}(x, y, z))==>\text { event }(\operatorname{sentRSU}(x, y, z)) \text {. }
\end{aligned}
$$

This query checks the authentication of a RSU towards a vehicle VE, namely, whenever the vehicle VE received a broadcast message that contains a RSU ID $x$, a timestamp $y$ and a RoadInfo record $z$, then this RSU really sent the broadcast message with the same $x, y$ and $z$.

For property Auth3, we define the following events:

1. event sentVEmRSU(spkey, id, lanesinfo, time).

2. event acceptedMRSU(spkey, id, lanesinfo, time).

The first event captures that a vehicle VE sent a message containing its ${ }_{645}$ short-term public key (of type spkey), the ID of the mRSU (of type id), the lane information (of type lanesinfo) and the timestamp (of type time) 8 Then, the query to verify is:

1. query $\mathrm{x}$ : spkey, $\mathrm{y}: \mathrm{id}, \mathrm{z}$ : laneid, w : time; event $(\operatorname{acceptedMRSU}(\mathrm{x}, \mathrm{y}, \mathrm{z}, \mathrm{w}))==>\operatorname{event}(\operatorname{sentVEmRSU}(\mathrm{x}, \mathrm{y}, \mathrm{z}, \mathrm{w}))$.

This query checks if whenever a mRSU accepted the message that contains a short-term public key $(x)$, the ID of the $\operatorname{mRSU}(y)$, lane information $(z)$, and 650 a timestamp $(w)$, then a vehicle really sent this freshly with $x, y, z$ and $w$.

To verify the integrity properties, we define an attacker process called pro$c$ AttInt for verifying the effect of message modification. For the property Int1, we specify a vehicle process, proc VE, such that when a vehicle receives a broadcast message from a RSU, and all the verification steps are successful, a piece

${ }_{655}$ of data named VERIFICATIONOK will be sent out on the channel $c$, otherwise, nothing will be sent, namely: free VERIFICATIONOK [private]. Here, VERIFICATIONOK is declared as a piece of data that is not available to the attackers at the beginning ([private]), but they can intercept it later.

Next, we specified the process procAttInt such that a modified version of the original broadcast message will be sent via the channel $c$. We examined different

\footnotetext{
${ }^{8}$ Note that the message sent by VE contains more elements, but only these four are used in the events as they are sufficient to uniquely identify the context of the communication.
} 
versions of message modifications, e.g., the same broadcast message signed with a different key, or when an element of the message is modified.

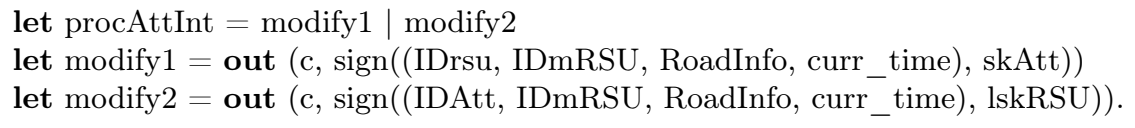

The case of property Int2 is similar to Int1, except that this time the process mRSU, procMRSU, sends out the "flag" VERIFICATIONOK on the successful. The process procAttInt this time sends different modifications of the original/correct traffic message on the channel $c$.

Finally, for the property Int3, both the processes proc $V E$ and proc $C A$ send out VERIFICATIONOK on the channel $c$ when the verification of their received messages are successful, and nothing otherwise. The process procAttInt sends different modifications of the two original messages, namely, the certificate request message from proc $V E$, and the certificate reply from proc $C A$.

In order to show that our protocol is capable of detecting the message modifications, we specified the following query in all three cases.

\section{query attacker(VERIFICATIONOK).}

This query checks if the attacker will be able to intercept the flag VERIFICATIONOK. As a result, in case of the original messages, ProVerif returned "RESULT not attacker(VERIFICATIONOK/I) is false", which means that the attacker was able to intercept the flag during the protocol, while for the modified versions of the original messages ProVerif returned "RESULT not attacker(VERIFICATIONOK/]) is true", as the verification of the messages was unsuccessful, hence, the flag was not sent out. We got the same result in cases when the other elements of the message were modified. Therefore, our proposed protocol is capable of distinguishing between a correct and a modified message.

\subsection{The security against insider attackers}

We assume that the mRSUs are well protected and therefore they are less vulnerable to attacks. Since in our approach, mRSUs are equipped with powerful $\mathrm{CPU}$ and resources, it can be assumed that intrusion detection systems, firewalls and anti-malware software are installed in them, and that they are physically well protected. Hence, in our analysis, compromised mRSUs are not considered.

\subsubsection{Compromised vehicles}

In the following, let us consider the settings in lines 21-23 of Algorithm 8 In order to generate a fake traffic congestion in a lane segment, say $\mathrm{L}_{1}$, an attacker has to affect the function increaseValue $\left(\mathrm{CV}\left(\mathrm{L}_{1}\right)\right)$, namely, increasing the congestion value of $\mathrm{L}_{1}$ that ultimately leads to increasing the duration for

695 the green light incorrectly. To do this, the attacker needs to compromise a certain number of vehicles (e.g., by infecting them with malware) and instruct them to send "Got Stuck" messages at a certain point of $\mathrm{L}_{1}$, even if they do not 
get stuck. Each compromised vehicle is instructed to generate and send a "Got Stuck" message in line 2 of Algorithm 3, and set their distance value (dist) to adjust them into the same point of the lane segment. Since the certificate and the signature will be valid, these messages will be accepted by the corresponding mRSU. However, because of the anomaly detection defined in Algorithm 9 . the attacker can only be successful if the number of benign vehicles sending "Not Stuck" messages (in that area and time) is less than the threshold $V_{a n o}^{\text {thres }}$.

705 Otherwise, the congestion value will not be increased. Hence, by setting the threshold to a small value we can limit the chance of the attacker.

Continuing the previous argument and scenario, let us assume that the number of benign vehicles is less than $V_{a n o}^{\text {thres }}$, so the attacker successfully increases the congestion value of $\mathrm{L}_{1}$. The attacker still cannot be sure that the mRSU

710 will set the traffic light program to give longer green time for $\mathrm{L}_{1}$, as this would also depend on the congestion values of the other lane segments. The attacker can achieve its goal if $\sum_{k}^{m} \mathrm{CV}\left(L_{j}\right)<\sum_{1}^{n} \mathrm{CV}\left(L_{j}\right)$ (line 30, Alg. 8), and this can be the case when either the total congestion value of the crossing lanes of $\mathrm{L}_{1}$ is very low or the attacker manages to increase the congestion value of $\mathrm{L}_{1}$ greatly

715 (e.g. when there are only compromised vehicles on $\mathrm{L}_{1}$ and there is no congestion on the crossing lanes). For this, the attackers need to control a huge amount of vehicles over a long time, which is costly and difficult.

\subsubsection{Compromised RSUs}

A compromised RSU can broadcast incorrect information about a road segment for the arriving vehicles. It can also remain silent not broadcasting anything, so the vehicles would not know which lane they arrived at, and hence the corresponding mRSU will not get any "Got Stuck" message from the vehicles?

1. In case a compromised RSU refuses to broadcast any message, the vehicles report to the corresponding $\mathrm{mRSU}$ the fact that it is not receiving any information (line 39 of $\mathrm{Alg}$. 4), so an engineer or system administrator can check and fix this RSU. However, this would require the availability of the satellite signal.

2. A compromised RSU can send incorrect lane information (LanesInfo in RoadInfo) with the result that the vehicles would include incorrect lane IDs into their messages. In order to mitigate the impact of this attack, a vehicle sends a message containing these incorrect lanes to the mRSU for verification purposes (line 2 and 10 of Alg. 3), which the mRSU verifies in lines 12-13 of Alg. 7 respectively. In case of inconsistency, the mRSU alerts the appropriate authority who will then fix the RSU. To check this in ProVerif, we declare the data FakeLanesInfo with the type lanesinfo, and define the process procComprRSU1 as follows.

\footnotetext{
${ }^{9}$ Vehicles could still send the "Got Stuck" messages but without the accurate information about the lane and location of the stuck vehicles, as we assumed that the SatNav system in the vehicles cannot identify accurately the lanes.
} 
1. free FakeLanesInfo : lanesinfo.

2. let procComprRSU1(lskRSU : lskey) =

3. let RoadInfo $=($ SatNvArea, NumLanes, FakeLanesInfo, DistInterval $)$ in

4. out (c, sign((IDrsu, IDmRSU, RoadInfo, curr_time), lskRSU)).

The record RoadInfo will be sent out on the channel $c$ with FakeLanesInfo (line 4). Then, we define the event acceptsMRSUFakeLanes(lanesinfo), which captures that the $m R S U$ accepts the message containing a data of type lanesinfo. Finally, the ProVerif query to check if the event when $m R S U$ accepts FakeLanesInfo would occur during the protocol run:

query event(acceptsMRSUFakeLanes(FakeLanesInfo)).

"RESULT not event(acceptsMRSUFakeLanes(FakeLanesInfo[J)) is true" was got as a result, which basically means that the mRSU never accepts the fake lane information by a compromised RSU. The reason is because the mRSU will carry out the verification on the correctness of the lane information (lines 12-13 and lines 20-21 Alg. 7).

3. Finally, a compromised RSU can broadcast a message with an incorrect mRSU ID, so the vehicles will send their messages to this incorrect mRSU. This attack can be prevented by the vehicles when they double check the received mRSUid against their MRSUrecord (see line 10 in Algorithm 2). In ProVerif, for the verification of this attack, we declare a fake mRSU ID called FakeIDmRSU, and define a process, procComprRSU2, which sends out a message containing this fake ID.

1. free FakeIDmRSU:id.

2. let procComprRSU2(lskRSU : lskey) =

3. let RoadInfo $=($ SatNvArea, NumLanes, LanesInfo $)$ in

4. out (c, sign((IDrsu, FakeIDmRSU, RoadInfo, curr_time), lskRSU)).

The query for this case is "query event(acceptsVEFakeMRSUID(id))", which captures that VE accepts the fake mRSU ID of type id. As a result, we got that this event only happens besides the attacker process procComprRSU2 when there is no SatNav signal in the area of the vehicle.

\section{Simulation}

We demonstrate the efficiency of our proposed approach compared to the traditional systems which rely on induction loops, cameras or purely static lights. 


\subsection{Simulation Environment}

We use the Veins simulation framework [24, which is an open source framework for vehicular networks and V2X simulations. Veins is built upon the SUMO simulator and the OMNET ++ discrete event simulator. For the simulation, Veins-5.0, OMNET ++5.4 .1 and SUMO 1.2.0 were used. We run the simulation on a selected area of the city of Preston, UK, which is exported from OpenStreetMap (see Fig. 5. ${ }^{10}$. As depicted in Fig. 5 , we set two routes for the cars (only cars were considered) to travel at the same time.
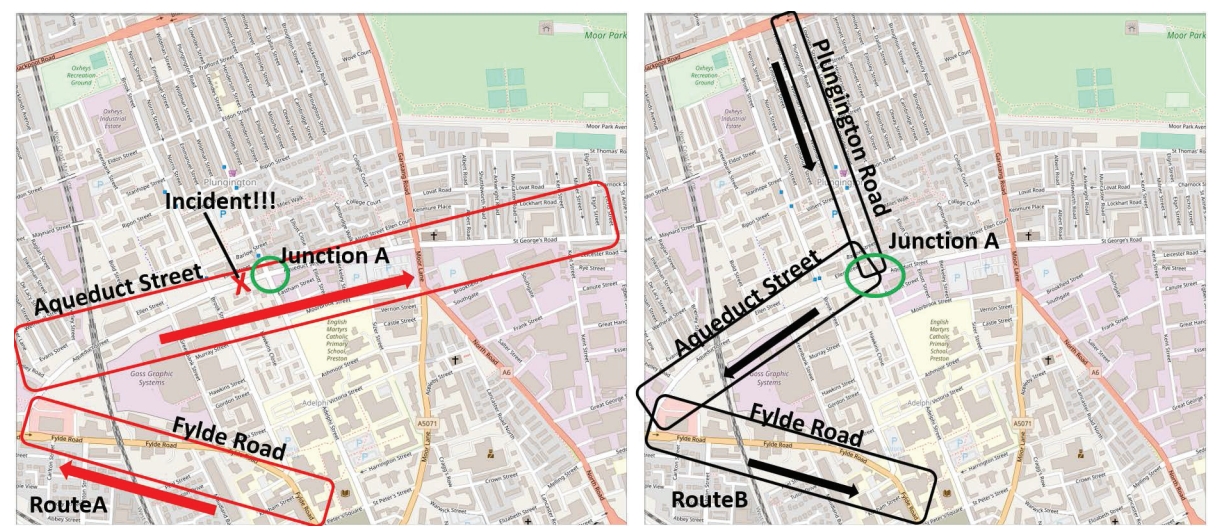

Figure 5: The simulation area, part of the city of Preston, UK, PR1 2TY. The two routes cross each other at junction A. RouteA starts from Fylde Road through Aqueduct street to the end, while on the right, RouteB starts from Plungington Road, through Aqueduct street and Fylde Road in the reverse direction compared to RouteA.

Aqueduct street has only two lanes in reverse directions, and in real life, a high number of cars or an incident on that road easily causes traffic congestion. In our simulation, we set an incident on RouteA (the car at the beginning of the flow breaks down) right before the junction A (illustrated by the red cross in Fig. 5p. Since at junction A, the cars from RouteB will turn into the road that has the incident, we expect a high delay for the cars on RouteA because 775 they have to wait until the flow from RouteB passes or until the traffic light at the junction A is green for RouteA in order to overtake the car broken down on the opposite lane. This situation is depicted in Fig. 6 .

We compare four different approaches based on the total travel time of all the cars on RouteA and RouteB from the start to the end of the simulation: The first approach is our proposed method based on V2I communication. The second approach is based only on an adaptive (actuated) traffic light concept with the car detectors installed beneath the roads. In this case, the traffic lights are able to prolong the green time favouring the road with a high number of

${ }^{10} \mathrm{We}$ choose Preston because it is the home of the first author's institution. 

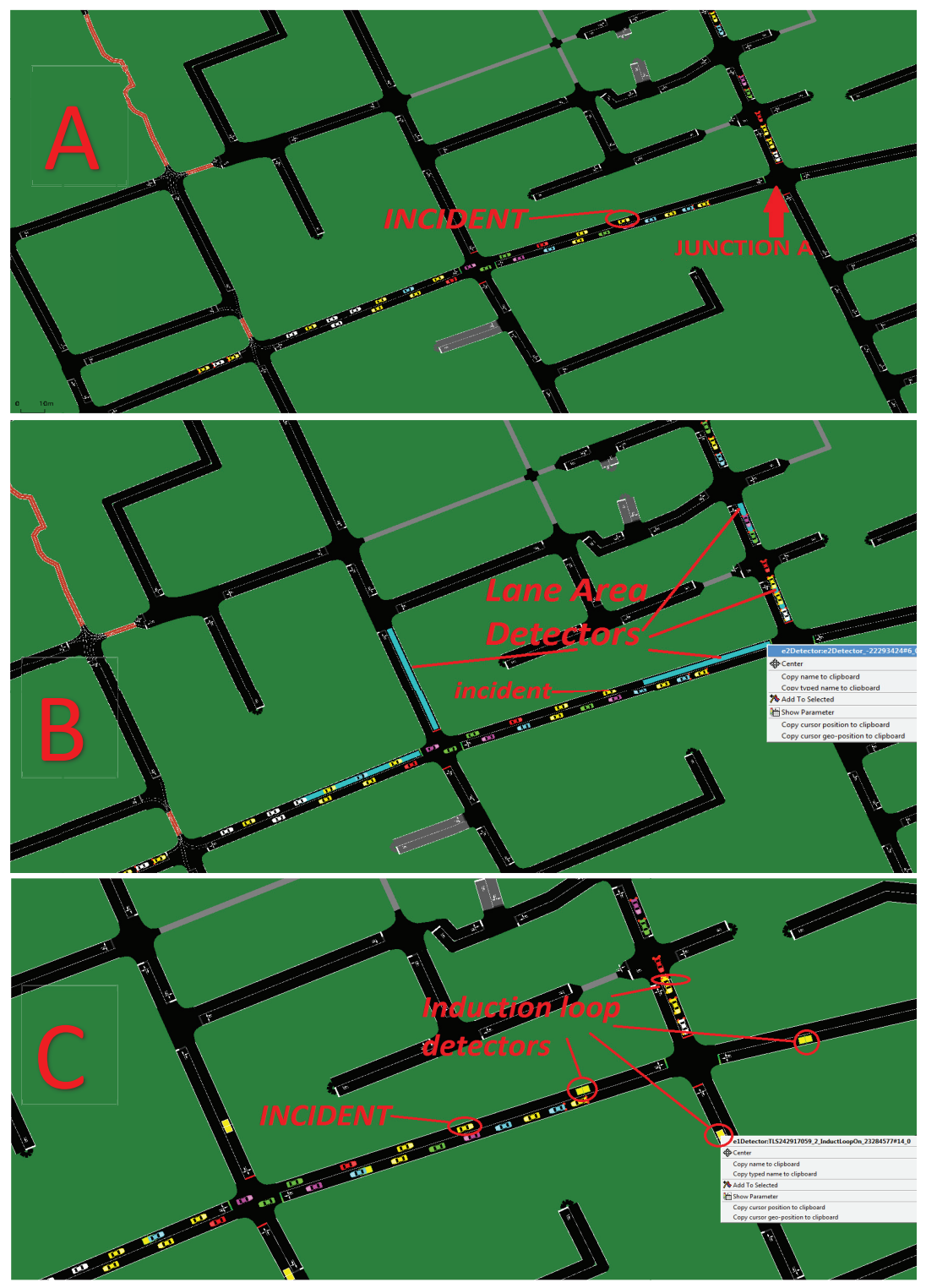

Figure 6: The simulation in Veins (SUMO) shows the car at the beginning of the flow on RouteA is broken down. Note that the cars travel on the left-side of the roads following the UK rules (a view from SUMO gui. A: Static, B:Lane area detector, C: Actuated).

cars following each other closely. The third is based on lane area detectors that

follows the concept of camcorders installed at the junctions to record and count the vehicles within a certain distance. The fourth approach is when only traffic 
lights with static program are used. Note that V2I communication is not applied in the last three cases.

The common simulation configurations we set in all four cases are:

790

- The car, map and route parameters and the car numbers are the same for all four cases. We used the default car following model implemented in SUMO, which is a modified version of the Krauss model by Stefan Krauss 42. The simulation time is set to $2500 \mathrm{sec}$, long enough for all the vehicles to be able to finish their journey.

- The first vehicle on RouteA is set to be broken down (speed is zero) at the middle of the road segment before junction A. This happens at time $165 \mathrm{~s}$ during the simulation of the V2I case, and 185s-190s during the simulation of the other three cases.

- RouteA and RouteB include five traffic light systems in total, each at a junction. Cars are allowed to overtake in the opposite lane. This makes it possible for the cars stuck behind the incident to overtake the car broken down on their opposite lane when there is a chance.

In the (purely) static lights case, the phases of the lights were set to $42 \mathrm{~s}$ (for the red-green light state) and 3s (for red-yellow), which is the default setting of 805 the map imported from OpenStreetMap.

In the actuated lights case, an induction detector loop is installed close to the junctions (Fig. 6/c) measuring the vehicle flow and extend the green time accordingly. We set the minimum duration of the red-green phase to $42 \mathrm{~s}$ that can be prolonged to the maximum duration of $63 \mathrm{~s}$ (i.e. $63 \mathrm{~s}=3 / 2 * 42 \mathrm{~s}$ ).

In case of the approach using lane area detector or camcorders installed at the junctions (Fig. 6/b) we set the length of the lane area detector such that the car broke down outside the sensing/detector area, but its sensing range is longer than a half of the road segments (enough to capture at least seven cars). In this case, camcorders cannot detect the cars that get stuck behind the incident.

In Veins, the V2X communication simulation is based on the $802.11 \mathrm{p}$ standard. We choose the following simulation configuration:

- The parameters of the 802.11p NIC include nic.mac1609_4.txPower = $20 \mathrm{~mW}$ for the transmission power, nic.mac1609_4.bitrate $=6 \mathrm{Mbps}$ bitrate level. The transmission power is set to strong enough for a car to be able to communicate with the mRSUs. Since we could not set the signal strength to accurately limit the communication to just one specific area, the mRSUs are programmed to ignore the messages sent from other areas.

- All the five traffic light systems on RouteA and RouteB are defined to be able to receive instructions from the mRSUs about the congestion status and change the light status accordingly. We also define five mRSUs to handle congestion status messages from vehicles in five different segments of RouteA and RouteB. 
- A vehicle will send a "Got Stuck" message to a corresponding mRSU if its speed drops to less than 1 for 21s (half of the length of the default light period, 42s). In order to reduce the amount of message lost due to channel congestion, each vehicle sets a random delay (with the uniform distribution) between 0 and $2 \mathrm{~s}$ before sending the message. Further, each vehicle also re-sends the same message (after the original one was already sent) once after a random delay between 0 and $2 \mathrm{~s}$. This delay period is small enough to avoid any change in the stuck status of a vehicle, and at the same time, can mitigate message lost during the simulation.

- For each junction with traffic lights in RouteA/RouteB (there are five of them in the simulation), the mRSUs check the congestion status on the crossing roads in its area after each second. If the number of cars that got stuck (i.e., speed $<1$ ) on one road (say, Road1) outweighs the number of the stuck cars on the crossing road (say, Road2), then the light system will be programmed to switch to green for Road1, red for Road2, and vice versa. Otherwise, if there is an equal number of stuck cars on the two crossing roads then the light system switches back to a default static program (i.e., $42 \mathrm{~s}$ for green-red, and 3s for yellow-red).

- A re-sent "Got Stuck" /"Go Again" message by a car will be ignored by an mRSU unless it has not received the original message due to packet lost.

- When the speed of a stuck car becomes greater than 1 for at least 20s (or a car moves into the area of a different mRSU), it will send a "Go Again" status message to the mRSU (that controls the area where it got stuck) with a random (uniform) delay between 0 and 2 . This is then re-sent once with a random delay between 0 and $2 \mathrm{~s}$ (after the original was sent).

Finally, the configuration for the lane area detection method is as follows:

- Since there are only cars on RouteA and RouteB, only the lane area detectors on those roads are defined. The lane area detectors are set to be longer than a half of the road segments, and be able to detect at least seven cars in its area (this is equivalent to a camcorder that is able to capture around half of a road segment).

- Static traffic lights are used by default with the same setting as in the other cases, namely, 42s for green-red, and 3s for yellow-red. Five mRSUs are defined to collect the information from the lane area detectors and change the traffic lights.

- Let RoadA and RoadB be two crossing roads at a junction: the traffic lights are set to green for RoadA and red for RoadB if the number of the vehicles detected by the lane area detector on $\operatorname{RoadA}$ is (at least) seven, and greater than the number of cars detected on RoadB for at least 20s, and vice versa. Otherwise, the traffic light is set to the default static phases. 
Table 8: TOTAL TRAVEL TIME COMPARISON 1

\begin{tabular}{|c|c|c||c|c|c|c|}
\hline $\begin{array}{c}\text { Cars } \\
\text { RouteA }\end{array}$ & $\begin{array}{c}\text { Cars } \\
\text { RouteB }\end{array}$ & $\begin{array}{c}\text { Incident } \\
\text { Duration }\end{array}$ & $\begin{array}{c}\text { V2I } \\
\text { (Our Approach) }\end{array}$ & $\begin{array}{c}\text { Lane Area } \\
\text { Detector }\end{array}$ & $\begin{array}{c}\text { Actuated } \\
\text { Lights }\end{array}$ & $\begin{array}{c}\text { Static } \\
\text { Lights }\end{array}$ \\
\hline \hline 12 & 14 & $280 \mathrm{~s}$ & 7690 & 8571 & 8969 & 9045 \\
\hline 24 & 14 & $280 \mathrm{~s}$ & 11709 & 13759 & 13922 & 14666 \\
\hline 36 & 14 & $280 \mathrm{~s}$ & 15732 & 19964 & 20442 & 21384 \\
\hline 48 & 14 & $280 \mathrm{~s}$ & 20522 & 26103 & 26376 & 27725 \\
\hline 60 & 14 & $280 \mathrm{~s}$ & 25692 & 31289 & 31917 & 33938 \\
\hline
\end{tabular}

Table 9: TOTAL TRAVEL TIME COMPARISON 2

\begin{tabular}{|c|c|c||c|c|c|c|}
\hline $\begin{array}{c}\text { Cars } \\
\text { RouteA }\end{array}$ & $\begin{array}{c}\text { Cars } \\
\text { RouteB }\end{array}$ & $\begin{array}{c}\text { Incident } \\
\text { Duration }\end{array}$ & $\begin{array}{c}\text { V2I } \\
\text { (Our Approach) }\end{array}$ & $\begin{array}{c}\text { Lane Area } \\
\text { Detector }\end{array}$ & $\begin{array}{c}\text { Actuated } \\
\text { Lights }\end{array}$ & $\begin{array}{c}\text { Static } \\
\text { Lights }\end{array}$ \\
\hline \hline 60 & 14 & $280 \mathrm{~s}$ & 25692 & 31289 & 31917 & 33938 \\
\hline 60 & 28 & $280 \mathrm{~s}$ & 35851 & 38460 & 40074 & 40715 \\
\hline 60 & 42 & $280 \mathrm{~s}$ & 43942 & 44392 & 46491 & 46778 \\
\hline 60 & 56 & $280 \mathrm{~s}$ & 50458 & 50744 & 52603 & 52778 \\
\hline 60 & 70 & $280 \mathrm{~s}$ & 56871 & 57251 & 59065 & 59283 \\
\hline
\end{tabular}

\begin{tabular}{|c|c|c||c|c|c|c|}
\hline $\begin{array}{c}\text { Cars } \\
\text { RouteA }\end{array}$ & $\begin{array}{c}\text { Cars } \\
\text { RouteB }\end{array}$ & $\begin{array}{c}\text { Incident } \\
\text { Duration }\end{array}$ & $\begin{array}{c}\text { V2I } \\
\text { (Our Approach) }\end{array}$ & $\begin{array}{c}\text { Lane Area } \\
\text { Detector }\end{array}$ & $\begin{array}{c}\text { Actuated } \\
\text { Lights }\end{array}$ & $\begin{array}{c}\text { Static } \\
\text { Lights }\end{array}$ \\
\hline \hline 60 & 70 & $280 \mathrm{~s}$ & 56871 & 57251 & 59065 & 59283 \\
\hline 60 & 70 & $380 \mathrm{~s}$ & 57659 & 60630 & 62482 & 64785 \\
\hline 60 & 70 & $480 \mathrm{~s}$ & 58748 & 64122 & 65391 & 67586 \\
\hline 60 & 70 & $580 \mathrm{~s}$ & 59090 & 65970 & 68769 & 69434 \\
\hline 60 & 70 & $680 \mathrm{~s}$ & 59616 & 67468 & 69215 & 71077 \\
\hline
\end{tabular}

\subsection{Simulation Results}

We run the simulation with the above configurations besides different numbers of cars on RouteA and RouteB. In order to see how the number of the cars behind the incident affects the total travel time, in Table 8 we increase the number of the cars on RouteA from 12 to 60, while the number of cars on RouteB is set to 14 in every case. We can see in Table 8 that the proposed

${ }_{875}$ V2I communication method has the lowest total travel time among the four methods. The reason is that once the car flow on RouteB passes the place of the incident, the mRSUs change all the five traffic light systems (at five junctions in RouteA/B) to green for the car flow on RouteA. The lane area detector method out performs the induction loop method and, unsurprisingly, the option with purely static lamps performs the worst. Finally, we can also see that by increasing the number of cars on RouteA, the time difference becomes greater.

In Table 9, we fix the number of cars on RouteA to 60, and change the cars on RouteB from 14 to 70 . With this, we want to check how the cars on the crossing road slow down the traffic on RouteA, preventing the cars stuck

${ }_{885}$ behind the incident from overtaking in the opposite lane. We can still see that the proposed V2I method out performs the other three, but (unlike the first comparison) the more cars on RouteB, the smaller the difference. We believe that our V2I method achieves a lower travel time compared to the lane area detection method because the lane area detectors are not be able to detect the incident outside the range, and hence, there is a delay until at least seven cars 
manage to overtake the broken down car and get into the range of the detectors due to the heavy traffic in the opposite lane. Finally, the reason the difference is decreasing with the increasing number of cars on RouteB is because the cars on RouteA and RouteB compete with each other by sending the "Got Stuck" messages to the mRSU. By increasing further the number of cars on RouteB the V2I remains the best among the four approaches. Eventually, Table 10 outlines the differences in case the incident duration is increased from 280s to 680s.

\subsection{Simulation discussion}

We have shown that besides the default parameters of the traffic lights programs downloaded from OpenStreetMap, there exists a configuration for our V2I solution that outperforms the other traditional solutions. Obviously, we could modify the light programs of the traditional methods, so that the actuated light can prolong the green time much longer than $63 \mathrm{~s}$, or use longer lane area detector to capture more cars. In this case, the parameter for the V2I

905 approach could be adjusted accordingly, for instance, the time period for sending a "Got Stuck" message could be shortened. Nevertheless, since in the V2I approach the mRSU can get a full picture of the congestion in lane segments, a more informed decision can be made than with the traditional systems.

\section{Message Overhead and Beacon Congestion}

In the following, we refer the reader to different points of Table 3. The size of the "Got Stuck" message of a vehicle in points 12-13 is estimated to be around 189 bytes besides the ECDSA signature scheme (with a 28 bytes key), and a 6 lane road. Specifically, 189 bytes = lanes_info (30 bytes) ${ }^{11}+$ loc_of_stuck $(6$ bytes $)+$ curr_time ( 8 bytes $)+$ state and Vtype (1 byte $)+$ mRSUid (3 bytes) + ECDSA signature and certificate (140 bytes [31]).

The signed "Not Stuck" message (points 4-5) is estimated to be around 190 bytes for a 6-lane road, namely, 190 bytes = lanes_info (30 bytes) + curr_time ( 8 bytes $)+$ curr_loc ( 8 bytes $)+$ state $(1$ byte $)+$ mRSUid ( 3 bytes $)+$ ECDSA signature and certificate (140 bytes).

920 Similarly, the estimated size of the signed "Go Again" (points 14-15) message is around 154 bytes $(6+8+140)$. The encrypted certificate request message (point 22 ) is between 84 and 256 bytes ${ }^{12}$, and finally, the signed "No Br" message (points 24-25) is estimated to be 153 bytes.

Eventually, the signed RSU broadcast in point 2 is around 163 bytes (where the plaintext is 23 bytes and the signature is 140 bytes). These values are in line with the literature (e.g. [31]) as they estimated the security messages to be around 200-400 bytes.

\footnotetext{
${ }^{11}$ In Table 2 Pavements is 1 byte, lane ID is 4 bytes, the direction information and lane width is 1 byte all together. Hence, the information for one lane is 5 bytes together.

1284 bytes can be in case of elliptic curve integrated encryption scheme (ECIES), while $128 / 256$ bytes in case of RSA.
} 
Table 11: COMPARING AVG MAC BUSY TIME AND THE MESSAGES RECEIVED BY EACH mRSU (1 accident, 280s accident duration). The percentage shows the amount of messages received by a mRSU out of the total messages addressed to it. This rate includes the re-sent "Got Stuck" and "Go Again" messages. The packet size was set to 200bytes.

\begin{tabular}{|c|c|c|c|c|c||c|c|}
\hline $\begin{array}{c}\text { MAC busy } \\
\text { time AVG }\end{array}$ & $\begin{array}{c}\text { mRSU }_{0} \\
\text { recvd }\end{array}$ & $\begin{array}{c}\text { RRSU }_{1} \\
\text { recvd }\end{array}$ & $\begin{array}{c}\text { mRSU }_{2} \\
\text { recvd }\end{array}$ & $\begin{array}{c}\text { mRSU }_{3} \\
\text { recvd }\end{array}$ & $\begin{array}{c}\text { RRSU }_{4} \\
\text { recvd }\end{array}$ & $\begin{array}{c}\text { Cars } \\
\text { RouteA }\end{array}$ & $\begin{array}{c}\text { Cars } \\
\text { RouteB }\end{array}$ \\
\hline \hline 0.193 & $72.5 \%$ & $80.6 \%$ & $85.7 \%$ & $100 \%$ & $83.3 \%$ & 50 & 50 \\
\hline 0.181 & $72.3 \%$ & $79.7 \%$ & $87 \%$ & $100 \%$ & $84 \%$ & 50 & 42 \\
\hline 0.144 & $74 \%$ & $78.4 \%$ & $90.3 \%$ & $100 \%$ & $81.4 \%$ & 50 & 28 \\
\hline 0.097 & $78.4 \%$ & $79.8 \%$ & $89.4 \%$ & $100 \%$ & $80.6 \%$ & 50 & 14 \\
\hline
\end{tabular}

To calculate the worst-case transfer rate, let us distinguish the cases with congestion and without congestion. In the first case, let us assume that 50 cars got stuck on RoadA and 50 cars on RoadB at juction A at the same time (we recall Fig. 6 and assume that a road segment can accommodate 50 cars). Unlike other studies (e.g. 31]), in our case, a vehicle does not process any message from other vehicles but a mRSU. Hence, we focus on the messages sent to a mRSU by the vehicles. Suppose that a vehicle sends ten "Got Stuck"/"Go Again" messages per second, in the worst-case scenario the corresponding mRSU would receive 1000 messages (10 messages x 100 cars). Based on the message overhead above, 189 bytes, this results in a transfer rate of $(1000 \times 189 \mathrm{byte}) / \mathrm{sec}$, which is around $1.51 \mathrm{Mbps}$. The rate would change to $1.6 \mathrm{Mbps}$ in case of $200 \mathrm{byte}$ messages, and $0.15 \mathrm{Mbps}$ in case each vehicle only sends one message in one second.

940 In case there is no congestion on RoadA and RoadB at all, each vehicle only sends one "Not Stuck" message of 190 bytes within a road segment, which in the worst-case would require 100 (cars) x 190byte, around $0.15 \mathrm{Mbps}$.

These are within $6 \mathrm{Mbps}$, the (default) transfer rate in DSRC 43]. Obviously, the actual rate would be less in practice as depending on when the cars got stuck, messages are sent at different times, and the "Not Stuck" messages are sent at different times as well. The simulations show the average MAC busy time in the first column of Table 11 .

Regarding channel congestion, during the simulation we adapted the IEEE 1609.4 multi-channel operation. In our case, however, there are no WSA (Wave

950 Service Announcement) beacons, as there is only one service. Both the RSUs and the vehicles only send WSM (Wave Short Messages) on the control channel $(\mathrm{CCH})$. Based on the scenario and the settings given in Section 6 and packet size of 200bytes, Table11 depicts the comparison of the average MAC busy time (for all cars in the simulation), and the amount of "Not Stuck", "Got Stuck" and "Go Again" messages received by each mRSU. The percentage in the case of $\mathrm{mRSU}_{0}$ is the lowest because it is in the area of the incident, hence, directly affected by the congestion. $\mathrm{mRSU}_{1}$ and $\mathrm{mRSU}_{2}$ receive the messages from the road segments next to the one with the incident, while in the area of $\mathrm{mRSU}_{3}$ there is no road congestion at all, hence, each car only sends one "Not Stuck" message (with a random delay), resulting in no message lost. Finally, $\mathrm{mRSU}_{4}$ covers the entire area on the right of junction A (Fig. 5).

Note that this percentage includes the re-sent "Got Stuck" and "Go Again" 
messages. In the simulation, after one re-transmission with a random delay, eventually all the "Got Stuck" and "Go Again" messages arrived at the mRSUs.

Moreover, in our system the vehicles only start sending traffic status messages to the mRSUs after it has arrived at the new road segment and already received the RSU broadcast. This limits the chance of collision between messages sent by a vehicle and a RSU. The RSUs in the neighbouring road segments are set to start broadcasting at different times to reduce message collision.

Finally, the authors in 44] showed that the delivery delay for safety messages increases linearly with the speed, both in case of DSRC (from $90 \mathrm{~ms} / 20 \mathrm{mph}$ to $130 \mathrm{~ms} / 50 \mathrm{mph})$ and LTE $(2690 \mathrm{~ms} / 20 \mathrm{mph}$ to $3000 \mathrm{~ms} / 50 \mathrm{mph})$. While we use different message contents, the result in this paper can be used as a reference.

\section{Discussion}

Security, privacy and accountability: Our approach also provides protection against physical attacks, such as intrusion into the on board devices of a vehicle or a $\mathrm{RSU} / \mathrm{mRSU}$ to extract the private keys. The hardware security modules (HSMs) or the trusted platformed modules (TPM) are designed to physically protect the private keys. Alternatively, we can use the on-demand secure key

980 generation approach in [45]. Denial of Services (DoS) attacks can be launched against our system, when the attackers send a huge amount of messages to the mRSUs. Although these messages are invalid, they consume the computing resource of the mRSUs. Known methods against DoS attacks [46, 47, can be implemented at the mRSUs to mitigate their impact. Finally, a jamming attack is always a potential threat in wireless communication, where the attacker prevents the RSUs, vehicles and mRSUs from receiving any messages. Different anti-jamming techniques 48 could be adopted to mitigate the effect of jamming.

From a privacy perspective, we assume that the vehicles regularly change their short-term public and private key pairs before arriving at a new road seg-

990 ment, and no future key pairs can be linked to the old ones (except for the trusted CA). This makes it difficult for an attacker or a system administrator/technician who eavesdrops the wireless communication to track the vehicles in the long-term. It is, however, still possible for someone to track the vehicles within a single road segment. Note that we adapted the concept of the pseudonyms because it provides a sensible balance between anonymity and accountability. However, our concept can be easily incorporated with different privacy enhancing approaches, e.g. group signature 49]. As for accountability, whenever there is an incident that requires an investigation (e.g. police or insurance company), our approach allows the CA to assist the investigation (under legislation) by revealing the link between the short-term public keys and the long-term ID of a vehicle.

The installation cost: Since the main RSUs require powerful computing and memory resources, their installation can be expensive. However, this can be adjusted by choosing how large a region a mRSU can control. The larger the 1005 region, the less mRSUs are required as only one mRSU is required for a region. In the case of the RSUs, since their numbers are much higher than the mRSUs, 
it is important to keep their cost reasonable. Each RSU only stores a minimal amount of data, and carries out two types of operations, signature generations and broadcasting. To moderate the cost of a RSU, a TPM is used to handle the cryptographic operations, which are much cheaper than using HSMs ${ }^{13}$

HSMs are powerful cryptoprocessors that perform cryptographic operations very effectively, and provide superior physical protection with tamper-resistant, tamper-proof properties. The private keys are generated inside the HSMs, and never leave it. Whilst very secure, HSMs are expensive and widely used by

1015 banks and financial institutions. Example HSMs include the IBM 4768 and 4769 PCIe ${ }^{14}$. TPMs are micro-controllers that stores keys, and digital certificates. The early version 1.2 of TPMs was typically integrated to the motherboard of a PC with cryptographic operations. Recently, TMP 2.0 has been improved to support a more extensive set of cryptographic operations including digital signature [50]. Compared to HSMs which cost thousands of Euros, TMPs are much cheaper (normally, less than 100 Euros).

V2V versus V2I concept: An alternative smart traffic control system could rely (entirely) on vehicle-to-vehicle $(\mathrm{V} 2 \mathrm{~V})$ communication as well, where vehicles will send traffic information to each other and a vehicle is able to set the traffic

1025 lights. While this concept can be cheaper (a vehicle would take over the tasks of our mRSU), it also poses a higher security risk, because vehicles can be more easily compromised than the mRSUs in our approach. Besides, we get back to the question of an accurate satellite navigation system, as if a concept is reliant only on $\mathrm{V} 2 \mathrm{~V}$ communication, then each vehicle needs to be able to accurately determine the lanes. Channel congestion is a well-known problem of V2V.

Lane identification: Identifying lanes is relevant for lane level traffic management, for example, traffic diversion could be done on time to avoid congestion or accidents on the motorways. Approaches to identifying lanes include camera based, e.g. [51, precise point positioning (PPP), e.g. [52, and GNSS shadow matching based methods, e.g. [36. The main challenge faced by camera based methods is their effectiveness in bad weather or at night, while the latter two approaches require the availability of the satellite signals (from several satellites at the same time), as well as depending on the surrounding obstacles such as buildings, mountains, trees etc.

$5 G$ technology: In this paper, we mainly focused on V2I communications (e.g., DSRC/802.11p). Our concept could be adapted in a 5G network, where instead of contacting the mRSUs the vehicles can follow the cellular V2X standard to communicate with the base station instead. Since $5 \mathrm{G}$ allows a much bigger message size, more information (e.g., pictures, videos) could be shared among the participants. RSUs could broadcast a set of pre-taken pictures about the road segments with the lane information and lane lines in different weather

\footnotetext{
${ }^{13}$ Note that it would be cheaper if the RSUs broadcast unsigned messages. However, anyone would be able to send a fake RoadInfo to the vehicles, and this cannot be detected in the areas where the satellite signal is permanently weak/unavailable.

${ }^{14}$ https://www.ibm.com/security/cryptocards/hsms. Accessed 01/07/2020.
} 
conditions and time to help vehicles identify the lanes better. Our message contents could be adapted in the cellular V2X standard. It is a potential future work to explore this in more detail.

\section{Conclusion and Future Work}

In this paper, we have provided an automated traffic congestion detection and notification concept based on V2I communication, including a system architecture and communication protocols. Unlike the previous studies and projects in a similar area, our solution is specifically designed for traffic congestion detection, hence it is different from those studies. The security properties of the new system were formally proved using the ProVerif tool. The security verification showed that our method is secure against external attackers, and in case of sufficient satellite signal, it is also secure against the compromised vehicles or RSUs. Finally, we demonstrate the performance of our concept through simulations with the Veins framework. Results have shown that besides the default settings OpenStreetMap, the total travel time of the traditional traffic control solutions is higher than the total travel time in our method (besides a reasonably chosen set of parameters and roads as an example).

\section{Acknowledgement}

This work was supported by the Ariel Cyber Innovation Center in conjunction with the Israel National Cyber directorate in the Prime Minister's Office.

\section{References}

[1] B. De Schutter, B. De Moor, Optimal traffic light control for a single intersection, European Journal of Control 4 (3) (1998) 260-276.

[2] B. Zhou, J. Cao, X. Zeng, H. Wu, Adaptive traffic light control in wireless sensor network-based intelligent transportation system, in: IEEE 72nd Vehicular Technology Conference (VTC 2010-Fall), 2010, pp. 1-5.

[3] F. Mehboob, M. Abbas, R. Jiang, R. A., A. S. Khan, S. Rehman, Trajectory based vehicle counting and anomalous event visualization in smart cities, Cluster Computer 21 (2018) 443-452.

[4] C. Osorio, K. Nanduri, Urban transportation emissions mitigation: Coupling high-resolution vehicular emissions and traffic models for traffic signal optimization, Transportation Research Part B: Methodological 81 (P2) (2015) 520-538.

[5] S. Djahel, M. Salehie, I. Tal, P. Jamshidi, Adaptive traffic management for secure and efficient emergency services in smart cities, in: The IEEE Pervasive Computing and Communication (PerCom) conference (WiP track), San Diego, California, USA, 2013. 
[6] I. Leontiadis, G. Marfia, D. Mack, G. Pau, C. Mascolo, M. Gerla, On the effectiveness of an opportunistic traffic management system for vehicular networks, IEEE Transactions on Intelligent Transportation Systems 12 (4) (2011) 1537-1548.

[7] D. Curiac, C. Volosencu, Urban traffic control system architecture based on wireless sensor-actuator networks, in: 2nd International Conference on Manufacturing Engineering, Quality and Production Systems, 2010, pp. 259-263.

[18] Scats (sydney coordinated adaptive traffic system), http://www.scats. com. au/, accessed: 23/03/2020.

[9] D. I. Robertson, R. D. Bretherton, Optimizing networks of traffic signals in real time-the scoot method, IEEE Transactions on Vehicular Technology 40 (1) (1991) 11-15.

[10] R. Chandra, C. Gregory, Insync adaptive traffic signal technology: Realtime artificial intelligence delivering real-world results, InSync White paper, accessed: 23/03/2020 (3 2012).

[11] I. Khan, K. Umar, H. Muhammmad, S. Bilal, S. B. Shah, Traffic density based light control system, Journal of American Academic Research 5 (2017) 90-94.

[12] M. J. Deshmukh, C. N. Modi, Designing an adaptive vehicular densitybased traffic signal controlling system, in: P. K. Sa, S. Bakshi, I. K. Hatzilygeroudis, M. N. Sahoo (Eds.), Recent Findings in Intelligent Computing Techniques, Springer Singapore, Singapore, 2018, pp. 107-115.

[13] A distributed image-retrieval method in multi-camera system of smart city based on cloud computing, Future Generation Computer Systems 81 (2018) $244-251$.

[14] J. Barthélemy, N. Verstaevel, H. Forehead, P. Perez, Edge-computing video analytics for real-time traffic monitoring in a smart city, Sensors 19 (9) (2019) (2019) 1 - 23.

[15] L. Caltagirone, M. Bellone, L. Svensson, M. Wahde, Lidar-camera fusion for road detection using fully convolutional neural networks, Robotics and Autonomous Systems 111 (2019) 125 - 131.

[16] C. Häne, L. Heng, G. H. Lee, F. Fraundorfer, P. Furgale, T. Sattler, M. Pollefeys, 3d visual perception for self-driving cars using a multi-camera system: Calibration, mapping, localization, and obstacle detection, Image and Vision Computing 68 (2017) $14-27$.

[17] T. Jeske, Floating car data from smartphones: What google and waze know about you and how hackers can control traffic, 2013. 
[18] K. Zetter, Hackers can mess with traffic lights to jam roads and reroute cars, accessed: 23/03/2020 (2014).

URL https://www .wired.com/2014/04/traffic-lights-hacking/

1125

[21] M. B. Sinai, N. Partush, S. Yadid, E. Yahav, Exploiting social navigation, CoRR abs/1410.0151 (2014).

[22] F. Kargl, P. Papadimitratos, L. Buttyan, M. Müter, E. Schoch, B. Wiedersheim, T. Thong, G. Calandriello, A. Held, A. Kung, J. Hubaux, Secure vehicular communication systems: implementation, performance, and research challenges, IEEE Communications Magazine 46 (11) (2008) 110-118.

[23] Proverif: Cryptographic protocol verifier in the formal model, http:// prosecco.gforge.inria.fr/personal/bblanche/proverif/, accessed: $23 / 03 / 2020$.

[24] C. Sommer, R. German, F. Dressler, Bidirectionally Coupled Network and Road Traffic Simulation for Improved IVC Analysis, IEEE Transactions on Mobile Computing 10 (1) (2011) 3-15. doi:10.1109/TMC.2010.133.

[25] V. Ta, A. Dvir, Y. Arie, Securing road traffic congestion detection by incorporating v2i communications, in: 2018 IEEE 19th International Symposium on "A World of Wireless, Mobile and Multimedia Networks" (WoWMoM), 2018, pp. 1-6.

[26] A. Laszka, B. Potteiger, Y. Vorobeychik, S. Amin, X. D. Koutsoukos, Vulnerability of transportation networks to traffic-signal tampering, 2016 ACM/IEEE 7th International Conference on Cyber-Physical Systems (ICCPS) (2016) 1-10.

[27] Q. A. Chen, Y. Yin, Y. Feng, Z. Mao, H. Liu, Exposing congestion attack on emerging connected vehicle based traffic signal control, in: Network and Distributed Systems Security (NDSS), 2018, pp. 1-15.

1155

[28] A. Burg, A. Chattopadhyay, K.-Y. Lam, Wireless communication and security issues for cyber-physical systems and the internet-of-things, Proceedings of the IEEE 106 (2018) 38-60.

[29] F. Harrou, A. Zeroual, Y. Sun, Traffic congestion monitoring using an improved knn strategy, Measurement 156 (2020) 107534. 
1160

[30] P. Xie, T. Li, J. Liu, S. Du, X. Yang, J. Zhang, Urban flow prediction from spatio-temporal data using machine learning: A survey, Information Fusion 59 (2020) $1-12$.

[31] M. Raya, J.-P. Hubaux, Securing vehicular ad hoc networks, Journal of Computer Security 15 (1) (2007) 39-68.

[32] J. R. Douceur, The sybil attack, in: Revised Papers from the First International Workshop on Peer-to-Peer Systems, IPTPS'01, Springer-Verlag, Berlin, Heidelberg, 2002, pp. 251-260.

[33] P. Goodwin, The economic costs of road traffic congestion, University College London, The Rail Freight Group, accessed: 23/03/2020 (2004).

[34] F. Ran, Z. Jiang, M. Xu, Vision-based lane detection algorithm in urban traffic scenes, in: K. Li, Y. Xue, S. Cui, Q. Niu (Eds.), Intelligent Computing in Smart Grid and Electrical Vehicles, Springer Berlin Heidelberg, Berlin, Heidelberg, 2014, pp. 409-419.

[35] P. Papadimitratos, L. Buttyan, T. Holczer, E. Schoch, J. Freudiger M. Raya, Z. Ma, F. Kargl, A. Kung, J.-P. Hubaux, Secure Vehicular Communication Systems: Design and Architecture, IEEE Communcations Magazine 46 (11) (2008) 100-109.

[36] A. D. R. Yozevitch, B. Ben-Moshe, Gnss accuracy improvement using rapid shadow transitions, IEEE Transactions on Intelligent Transportation Systems 15 (3) (2014) 1113-1122.

[37] A. Boualouache, S. Senouci, S. Moussaoui, A survey on pseudonym changing strategies for vehicular ad-hoc networks, IEEE Communications Surveys Tutorials 20 (1) (2018) 770-790.

[38] M. Abadi, B. Blanchet, C. Fournet, Just fast keying in the pi calculus, ACM Transactions on Information and System Security (TISSEC) 10 (3) (2007) 1-59.

[39] B. Blanchet, Symbolic and computational mechanized verification of the ARINC823 avionic protocols, in: 30th IEEE Computer Security Foundations Symposium (CSF'17), IEEE, Santa Barbara, CA, USA, 2017, pp. 68-82.

[40] B. Blanchet, A. Chaudhuri, Automated formal analysis of a protocol for secure file sharing on untrusted storage, in: Proceedings of the 29th IEEE Symposium on Security and Privacy (SP'08), IEEE, 2008, pp. 417-431.

[41] B. Blanchet, B. Smyth, V. Cheval, M. Sylvestr, Proverif 2.00: Automatic cryptographic protocol verifier, user manual and tutorial, User Manual (5 2018). 
[42] S. Krauß, Microscopic modeling of traffic flow: Investigation of collision free vehicle dynamics, Ph.D. thesis (1998).

[43] J. B. Kenney, Dedicated short-range communications (dsrc) standards in the united states, Proceedings of the IEEE 99 (7) (2011) 1162-1182.

[44] Vehicle-to-vehicle (v2v) and vehicle-to-infrastructure (v2i) communication in a heterogeneous wireless network - performance evaluation, Transportation Research Part C: Emerging Technologies 68 (2016) 168 - 184.

[45] S. N. Muthaiah, On-demand secure key generation in a vehicle-to-vehicle communication network, patent No. US8526606B2, Filed Dec. 20th., 2010, Issued Sept. 3rd., 2013 (September 2013).

[46] M. Mejri, J. Ben-Othman, Gdvan: A new greedy behavior attack detection algorithm for vanets, IEEE Transactions on Mobile Computing 16 (03) (2017) 759-771.

[47] D. Mansouri, L. Mokddad, J. Ben-othman, M. Ioualalen, Preventing denial of service attacks in wireless sensor networks, in: 2015 IEEE International Conference on Communications (ICC), 2015, pp. 3014-3019.

[48] A. Mpitziopoulos, D. Gavalas, C. Konstantopoulos, G. Pantziou, A survey on jamming attacks and countermeasures in wsns, IEEE Communications Surveys Tutorials 11 (4) (2009) 42-56.

[49] J. Guo, J. Baugh, S. Wang, A group signature based secure and privacypreserving vehicular communication framework, 2007, pp. 103-108.

[50] T. C. Group, Tcg pc client platform tpm profile (ptp) specification, TCG Published, accessed: 12/03/2020 (2015).

1220 URL https://www.trustedcomputinggroup.org/wp-content/uploads/

1. PC-Client-Specific-Platform-TPM-Profile-for-TPM-2-0-v43-150126. pdf

[51] A. Gupta, A. Choudhary, A framework for camera-based real-time lane and road surface marking detection and recognition, IEEE Transactions on Intelligent Vehicles 3 (4) (2018) 476-485. doi:10.1109/TIV.2018.2873902.

[52] V. L. Knoop, P. F. de Bakker, C. C. J. M. Tiberius, B. van Arem, Lane determination with gps precise point positioning, IEEE Transactions on Intelligent Transportation Systems 18 (9) (2017) 2503-2513. 


\section{Appendix A. Auxiliary Algorithms}

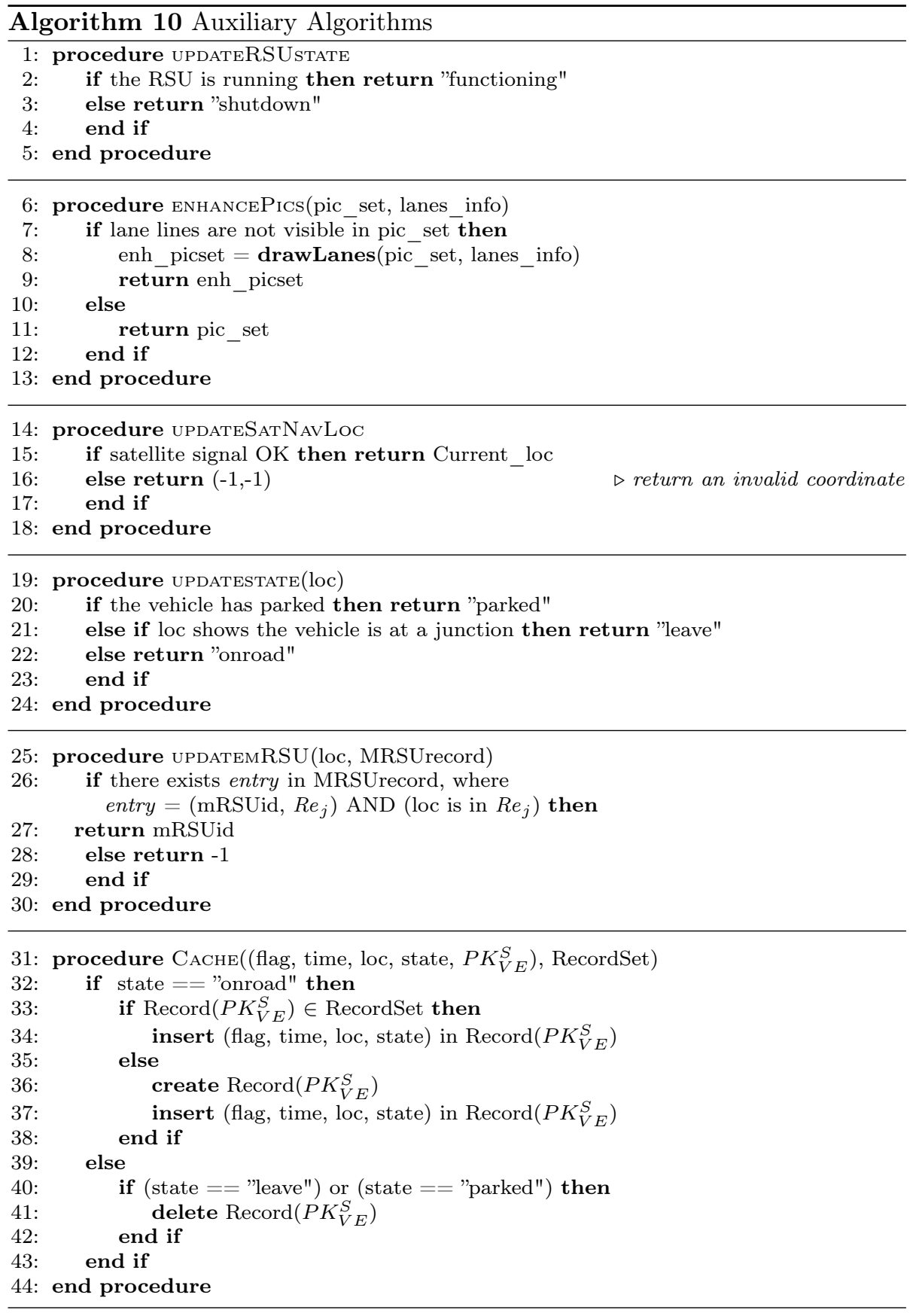

